Preprint typeset using $\mathrm{LAT}_{\mathrm{E}} \mathrm{X}$ style emulateapj v. 5/2/11

\title{
THE SHAPE OF THE INNER MILKY WAY HALO FROM OBSERVATIONS OF THE PAL 5 AND GD-1 STELLAR STREAMS
}

\author{
Jo Bovy ${ }^{1,2}$, Anita Bahmanyar ${ }^{1}$, Tobias K. Fritz ${ }^{3}$, And Nitya Kallivayalil ${ }^{3}$
}

\begin{abstract}
We constrain the shape of the Milky Way's halo by dynamical modeling of the observed phase-space tracks of the Pal 5 and GD-1 tidal streams. We find that the only information about the potential gleaned from the tracks of these streams are precise measurements of the shape of the gravitational potential - the ratio of vertical to radial acceleration - at the location of the streams, with weaker constraints on the radial and vertical accelerations separately. The latter will improve significantly with precise proper-motion measurements from Gaia. We measure that the overall potential flattening is $0.95 \pm 0.04$ at the location of GD $-1([R, Z] \approx[12.5,6.7] \mathrm{kpc})$ and $0.94 \pm 0.05$ at the position of Pal $5([R, Z] \approx[8.4,16.8] \mathrm{kpc})$. Combined with constraints on the force field near the Galactic disk, we determine that the axis ratio of the dark-matter halo's density distribution is $1.05 \pm 0.14$ within the inner $20 \mathrm{kpc}$, with a hint that the halo becomes more flattened near the edge of this volume. The halo mass within $20 \mathrm{kpc}$ is $1.1 \pm 0.1 \times 10^{11} \mathrm{M}_{\odot}$. A dark-matter halo this close to spherical is in tension with the predictions from numerical simulations of the formation of dark-matter halos.
\end{abstract}

Subject headings: dark matter - Galaxy: fundamental parameters - Galaxy: halo - Galaxy: kinematics and dynamics - Galaxy: structure - globular clusters: individual (Palomar 5)

\section{INTRODUCTION}

The Milky Way's gravitational potential within the inner tens of kpc is key to various important problems in galactic astrophysics and near-field cosmology. Containing the vast majority of all of its stars, hosting almost all of its building blocks, and being amenable to detailed observational characterization, this volume contains a wealth of information on the formation of the Galactic bulge and disk deep in the Milky Way's potential well, the formation channels of the stellar halo, and the detailed large- and small-scale structure of the dark matter halo and its response to the growth of the baryonic components. It is in this volume soon to be mapped in detail by the Gaia satellite (de Bruijne 2012) - that we can determine the Milky Way's mass and light budget (e.g., Flynn et al. 2006; Bovy \& Rix 2013; McKee et al. 2015), the dark-matter halo's shape and radial profile near and within its scale radius (e.g., Olling \& Merrifield 2000; Binney \& Piff 2015), and the abundance of lowmass substructure (e.g., Johnston et al. 2002; Ibata et al. 2002). Determining the three-dimensional force field is a prerequisite to any study of the orbital properties of stars and star clusters in the disk and halo (Binney 2013; Rix \& Bovy 2013).

The shape of the dark-matter halo is of particular interest and poorly constrained by current data. Because the primary constraint on the gravitational potential in the Milky Way and external galaxies alike has been the rotation curve, which is hardly sensitive to the detailed three-dimensional shape of a mass distribution, precise measurements of the shapes of galactic dark-matter ha-

1 Department of Astronomy and Astrophysics, University of Toronto, 50 St. George Street, Toronto, ON, M5S 3H4, Canada; bovy@astro.utoronto.ca

2 Alfred P. Sloan Fellow

3 Department of Astronomy, University of Virginia, Charlottesville, 3530 McCormick Road, VA 22904-4325, USA los are close to non-existent. This is an unfortunate situation, because numerical simulations of the formation of dark-matter halos make strong predictions for their three-dimensional shapes. In the absence of baryons, dark-matter halos are generally found to be strongly triaxial (e.g., Frenk et al. 1988; Dubinski \& Carlberg 1991; Warren et al. 1992; Cole \& Lacey 1996; Jing \& Suto 2002; Vera-Ciro et al. 2011). The growth of a galactic baryonic disk causes the halos to become more axisymmetric and aligned with the disk, but the minor-to-major axis ratio $c / a$ changes only by a few tenths to $c / a \approx 0.7$ to 0.8 (Dubinski 1994; Gustafsson et al. 2006; Debattista et al. 2008; Abadi et al. 2010; Kazantzidis et al. 2010). The amount of sphericalization depends on the mass of the baryonic component (Kazantzidis et al. 2010). Because the Milky Way's disk is close to maximal (Bovy \& Rix 2013; Wegg et al. 2016), the inner dark-matter halo is expected to have $c / a \approx 0.8$. This is an especially interesting prediction, as, e.g., non-canonical dark-matter models such as self-interacting dark matter predict that halos should be spherical in their high-density inner regions (Spergel \& Steinhardt 2000; Yoshida et al. 2000; Davé et al. 2001; Miralda-Escudé 2002; Peter et al. 2013).

In the Milky Way we can measure the threedimensional force (or, equivalently, acceleration) field and thus the halo's shape in different ways. Assuming that the inner stellar halo is in a quasi-stationary state, equilibrium modeling using the Jeans equations (e.g., Loebman et al. 2014; Bowden et al. 2016) or using the Jeans theorem (e.g., Binney \& Piffl 2015) can be used to determine the force field from the observed positions and velocities of halo stars. So far, this approach has had limited success due to the difficulty of observing the stellar halo's kinematics over a large enough volume and at high enough precision. Measurements of the shape of the inner halo $(r \lesssim 20 \mathrm{kpc})$ from these measurements range from strongly oblate $(c / a \approx 0.4$; Loebman et al. 
2014) to prolate (Bowden et al. 2016). The trajectories of hypervelocity stars are also sensitive to the shape of the force field and can in the future be used to constrain the dark-matter halo's shape (Gnedin et al. 2005).

The current best measurements of the shape of the Milky Way's gravitational potential in the halo region come from observations of stellar streams. Streams form when a satellite galaxy or a globular cluster gets tidally destroyed in the Milky Way's gravitational potential (Johnston 1998; Helmi \& White 1999; Tremaine 1999): mass loss due to tidal stripping leads to leading and trailing arms at slightly different orbital energies than the progenitor cluster. The track traced by the stellar stream is close to an orbit (Eyre \& Binney 2011; Sanders \& Binney 2013) and thus provides a rather direct measurement of the local acceleration near the stream. Dynamical modeling of tidal streams has made much progress in the last few years, with multiple distinct methods proposed for realistically approximating the formation and evolution of streams in general gravitational potentials (e.g., Eyre \& Binney 2011; Sanders \& Binney 2013; Varghese et al. 2011; Bovy 2014; Price-Whelan et al. 2014; Sanders 2014; Amorisco 2015; Fardal et al. 2015; Küpper et al. 2015). However, this progress in modeling has led to only a modest improvement in the observational modeling of tidal streams and determining the shape of the halo. Variants of particle-spray-type modeling, where the formation of the stream is simply modeled by orbit integration of a finite number of ejected stars, have been used to constrain the potential shape using the GD-1 (Grillmair \& Dionatos 2006) and Pal 5 streams (Odenkirchen et al. 2001). Bowden et al. (2015) used the data from Koposov et al. (2010) to determine the overall potential flattening $q_{\Phi}$ to be $q_{\Phi}=0.90_{-0.10}^{+0.05}$ in a simple logarithmic potential model. Küpper et al. (2015) employed a three-component disk-bulge-halo model to determine the shape of the halo's potential, finding $q_{\Phi}^{h} \approx 0.95_{-0.12}^{+0.15}$, but fixing the parameters describing the disk and bulge. The uncertainties in these measurements do not allow a stringent test with respect to the predictions from numerical simulations discussed above.

A second class of approaches uses action-angle coordinates to build simple models of tidal streams (Helmi \& White 1999; Tremaine 1999; Eyre \& Binney 2011; Bovy 2014; Sanders 2014). In particular, the simple modeling approach proposed in Bovy (2014) (hereafter B14) and Sanders (2014) allows one to make smooth, continuous predictions for the present-day structure of an observed stream. In this paper we present the first application of this technique to observational data, by modeling the Pal 5 and GD-1 streams. We use general models for the Milky Way's gravitational potential with a large amount of freedom to investigate the exact nature of the constraints on the potential provided by these streams. We demonstrate that even with the current data, these streams are exquisitely sensitive to the shape of the darkmatter halo and the overall shape of the potential.

The structure of this paper is as follows. We discuss our Milky-Way model and prior constraints on the shape of the dark-matter halo from existing data in $\S 2$. In $\S 3$ we present the details of our stream-fitting methodology. We then apply this methodology to observational data for Pal 5 in $\S 4$ and for GD-1 in $\S 5$, including com- parisons to previous fits in the literature. We combine our new measurements of the force-field near Pal 5 and GD-1 with constraints coming primarily from the rotation curve and vertical disk dynamics in $\S 6$ to make a determination of the axis ratio $c / a$ of the dark-matter halo's density distribution and discuss these results. We present our conclusions in $\S 7$.

\section{MILKY WAY MODEL AND PRIOR CONSTRAINTS}

\subsection{Milky Way model}

For most of the analyses in this paper we use a simple three-component potential model for the Milky Way consisting of a bulge, disk, and halo component. The bulge is in all cases modeled with a spherical power-law density profile that is exponentially cut-off. The power-law exponent is fixed to -1.8 and the exponential cut-off radius is set to $1.9 \mathrm{kpc}$. In most cases, we model the disk component as a Miyamoto-Nagai potential with three free parameters: the scale length $h_{R}$, the scale height $h_{z}$, and an amplitude parameter. We will also explore modeling the disk as a double exponential density profile in the radial and vertical directions, which has the same three free parameters.

The halo component is represented by a triaxial Navarro-Frenk-White (NFW) profile (Navarro et al. 1997) with constant density along similar, co-axial ellipsoids. In the frame $\left(x^{\prime}, y^{\prime}, z^{\prime}\right)$ aligned with the three symmetry axes we have that

$$
\rho^{h}\left(x^{\prime}, y^{\prime}, z^{\prime}\right)=\frac{M}{4 \pi r_{s}^{3}} \frac{1}{\left(m / r_{s}\right)\left(1+m / r_{s}\right)^{2}},
$$

where

$$
m^{2}=x^{\prime 2}+\frac{y^{\prime 2}}{(b / a)^{2}}+\frac{z^{\prime 2}}{(c / a)^{2}} .
$$

We also allow the orientation of the $\left(x^{\prime}, y^{\prime}\right)$ frame to be specified with respect to the Sun-Galactic-center line $(Y=0$ in the Galactocentric frame $(X, Y, Z))$, but keep $z^{\prime} \equiv Z$. The axis ratio $c / a$ then describes the flattening of the halo perpendicular to the Galactic disk and $b / a$ deviations from axisymmetry in the disk plane. We compute the gravitational potential and forces corresponding to this density using the expressions in Merritt \& Fridman (1996), performing the necessary integrals with 50-th order Gauss-Legendre quadrature. This potential-density pair as well as the generalization to arbitrary orientations between $(X, Y, Z)$ and $\left(x^{\prime}, y^{\prime}, z^{\prime}\right)$ has been implemented into galpy (Bovy 2015) for the purpose of this work.

There are few robust constraints on the axis ratio $b / a$ of the dark-matter halo in the Milky Way. If $b / a \neq 1$ in the inner Milky Way, the closed orbits in the disk around which stellar orbits librate would not be circular. This non-circularity can be detected using the chemical and kinematic properties of disk stars. The lack of azimuthal abundance differences in the few kpc near the Sun constrains $b / a$ to be near one or $y$ and $y^{\prime}$ to be close to aligned (Bovy et al. 2014). The kinematics of disk stars within a few kpc from the Sun strongly constraints $b / a$ to be close to one for any angle between $y$ and $y^{\prime}$ (Bovy et al. 2015).

Other constraints on $b / a$ come from tidal streams. A successful model of the tidal tails of the Sgr dwarf galaxy has been found with $b / a \approx 0.7$ (Law \& Majewski 2010), 

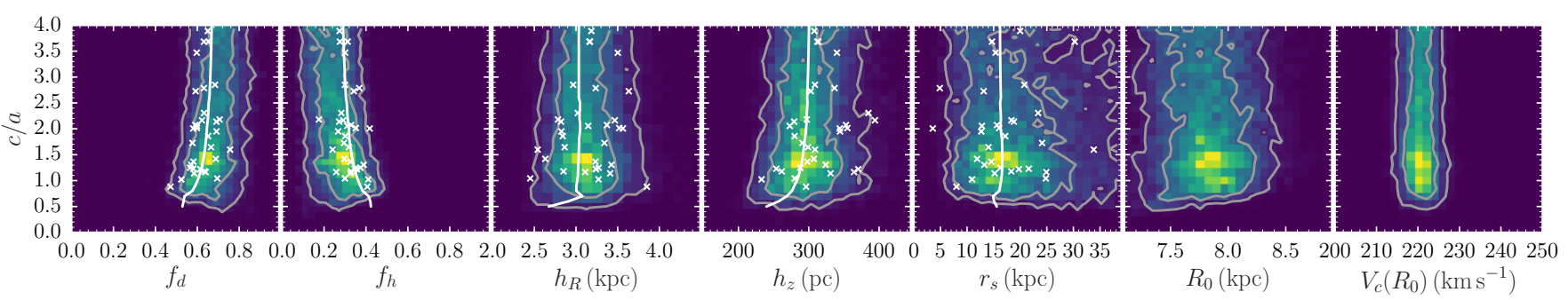

FIG. 1.- Constraints on the halo-axis ratio $c / a$ and the disk, bulge, and halo components of the Milky Way from dynamical data prior to the stream measurements from this paper. This figure displays the PDF of the eight parameters describing the Milky Way potential, focusing on the correlation between $c / a$ and the other parameters. The contours contain $68 \%$ and $95 \%$ of the distribution. The white line is the locus of the best-fit values of the parameters shown horizontally as a function of $c / a$ for $R_{0}=8 \mathrm{kpc}$ and $V_{c}\left(R_{0}\right)=220 \mathrm{~km} \mathrm{~s}{ }^{-1}$. The white crosses display the values of the fixed parameters for the 32 potential families used in the analysis of Pal 5 and GD-1; only the horizontal parameters are fixed in these families. The dynamical data prior to the Pal 5 and GD-1 stream data do not strongly constrain the halo axis ratio, except to rule out strongly flattened halo densities with $c / a \lesssim 0.7$ (2.5\% confidence).

but if this axis ratio is assumed in the inner $\approx 20 \mathrm{kpc}$, no model compatible with the observed Pal 5 tidal stream can be found (Pearson et al. 2015).

We can use the sensitivity to $b / a$ and $c / a$ of orbit segments compatible with the Pal 5 and GD-1 data described below as a proxy for how sensitive these data on the average location of the track are to these parameters. For both streams we find that such orbit segments are hardly sensitive to even large variations in $b / a(b / a$ going from $1 / 2$ to 2$)$, whether or not $(X, Y)$ and $\left(x^{\prime}, y^{\prime}\right)$ are aligned or not. The sensitivity to the maximally misaligned setup is for both streams larger than that of the aligned setup, but all changes in $b / a$ lead to orbit segments that differ by an order of magnitude less than orbits for different $c / a$. This is the case because the streams are located near a Galactocentric azimuth of zero (GD1) and $\pi$ (Pal 5), that is, both are approximately along the line connecting the Sun to the Galactic center.

The lack of sensitivity to changes in $b / a$ only apply to the stream track. Like Pearson et al. (2015), we find that if the halo is triaxial with $b / a \neq 1$ a narrow stream does not form at the phase-space location of Pal 5 because the orbital history of the stream is chaotic. Even though the orbit segments near the stream do not depend strongly on $b / a$, orbits compatible with the stream integrated for multiple Gyr are chaotic for $b / a \neq 1$ and the stream would therefore not be narrow. This fact could be used to set a limit on $|b / a-1|$ from the observed width of $\mathrm{Pal}$ 5 , but we do not pursue this in this paper.

Because observational data indicate that $b / a \approx 1$ and because both the Pal 5 and GD-1 stream tracks are largely insensitive to changes in $b / a$, we fix $b / a$ to one in what follows.

\subsection{Prior constraints on the halo axis ratio}

To determine what, if any, constraints on the halo axis ratio $c / a$ we can set based on existing dynamical data and to find a realistic set of potentials to use in the analysis of the Pal 5 and GD-1 streams below, we perform fits of the above potential model to a variety of dynamical data on the bulge, disk, and halo. This fit is similar to that used to arrive at the fiducial MWPotential2014 model of Bovy (2015) and we summarize the data, fit procedure, and differences with MWPotential2014 here.

The data are similar to those used by Bovy \& Rix (2013) and Bovy (2015):

a) The velocity dispersion $\sigma_{b}=117 \pm 15 \mathrm{~km} \mathrm{~s}^{-1}$ mea- sured in Baade's window (Dehnen \& Binney 1998; Binney \& Tremaine 2008);

b) The vertical force $\left|F_{Z}\right|=67 \pm 6\left(2 \pi G M_{\odot} \mathrm{pc}^{-2}\right)$ at the solar circle at $|z|=1.1 \mathrm{kpc}$ and the local visible surface density $\Sigma=55 \pm 5 M_{\odot} \mathrm{pc}^{-2}$ from Zhang et al. (2013);

c) The vertical force measurements at $|z|=1.1 \mathrm{kpc}$ of Bovy \& Rix (2013) (their Table 3);

d) The terminal-velocity measurements of Clemens (1985) and McClure-Griffiths \& Dickey (2007), modeled in the same way as in Bovy \& Rix (2013). In $\S 6$ we replace the older Clemens (1985) by the newer McClure-Griffiths \& Dickey (2016) data;

e) The mid-plane $(Z=0)$ density at the solar circle of Holmberg \& Flynn (2000): $\rho\left(R_{0}, Z=0\right)=0.10 \pm$ $0.01 M_{\odot} \mathrm{pc}^{-3}$;

f) The measurements of the logarithmic slope of the rotation curve from Bovy et al. (2012), represented in the same way as in equation (41) in Bovy \& Rix (2013);

g) The measurement of the total mass within $60 \mathrm{kpc}$ from Xue et al. (2008): $M(r<60 \mathrm{kpc})=4.0 \pm$ $0.7 \times 10^{11} M_{\odot}$.

Unlike Bovy (2015), we allow the Sun's distance to the Galactic center $R_{0}$ and the circular velocity $V_{c}\left(R_{0}\right)$ at $R_{0}$ to vary. Based on recent measurements of $R_{0}$ (Chatzopoulos et al. 2015; Bland-Hawthorn \& Gerhard 2016; Boehle et al. 2016), we use the constraint $R_{0}=$ $8.1 \pm 0.1 \mathrm{kpc}$. We also use the constraint $V_{c}\left(R_{0}\right)=$ $218 \pm 10 \mathrm{~km} \mathrm{~s}^{-1}$ (Bovy et al. 2012). We will see that all determinations of $c / a$ in this paper are uncorrelated with $R_{0}$ and $V_{c}\left(R_{0}\right)$, so these constraints are not crucial.

The free parameters of the potential model are the amplitude, scale length $h_{R}$, and scale height $h_{z}$ of the disk component, the amplitude, scale radius $r_{s}$, and axis ratio $c / a$ of the halo component, $R_{0}$, and $V_{c}\left(R_{0}\right)$. The amplitudes of the disk, halo, and bulge components are specified in terms of the fraction of the radial force at $(R, Z)=\left(R_{0}, 0\right)$ that they provide (with $f_{d}$ for the disk fraction and $f_{h}$ for the halo fraction). The results from fitting the data above are summarized in Figure 1. 
This figure displays the posterior probability distribution function (PDF) when modeling the disk using a Miyamoto-Nagai density, focusing on the correlation between $c / a$ and the other model parameters. The PDFs are similar when using a double-exponential disk, except that the scale length of the disk is $h_{R} \approx 2.4 \mathrm{kpc}$ (and $h_{R} \approx 2.2 \mathrm{kpc}$ when also including a gas component as discussed below, in agreement with Bovy \& Rix 2013).

It is clear from Figure 1 that there is only a weak constraint on $c / a$ from these data and that $c / a$ is largely uncorrelated with all of the other model parameters. However, the data disfavor strongly flattened halo densities, with only $2.5 \%$ probability that $c / a<0.7$ in the case of a Miyamoto-Nagai disk and that $c / a<0.8$ for a doubleexponential disk (both of these $c / a$ limits are about 0.2 smaller for $0.5 \%$ probability). Note that these models do not include a more extended gas component. Including such a component to the double-exponential disk model with a scale length that is twice that of the stellar component, a scale height of $150 \mathrm{pc}$, and a local surface density of $10 \mathrm{M}_{\odot} \mathrm{pc}^{-2}$, the $2.5 \%$ lower limit is 0.65 . The lower cut-off in $c / a$ in all of these models happens because the $F_{Z}$ data from Bovy \& Rix (2013) indicate a short scale length $(\approx 2.5 \mathrm{kpc})$ for the total mass within $\approx 1 \mathrm{kpc}$ of the disk mid-plane. The halo's effective scale length is $\gtrsim 4 \mathrm{kpc}$, thus making it difficult to satisfy the short mass scale length implied by the $F_{Z}$ measurements if too much of the halo's mass is concentrated near the mid-plane due to the halo's flattening. Above this lower cut-off on $c / a$, the $\mathrm{PDF}$ for $c / a$ is almost flat up to $c / a=4$, which is the highest $c / a$ that we consider.

Thus, the pre-stream dynamical data do not provide a strong constraint on $c / a$ beyond that it cannot be much smaller than one. We re-do this fit in $\S 6$ adding in the new measurements of the force field from the next sections.

\section{STREAM-FITTING METHODOLOGY}

The data on the Pal 5 and GD-1 stellar streams that we consider in the next two sections come in the form of measurements of the phase-space location of the track of these streams. That is, we have measurements of some combination of the sky position, distance from the Sun, proper motion vector, and line-of-sight velocity measured as a function of a coordinate going along the stream. For the Pal 5 stream we also have measurements of the sixdimensional position and velocity of its progenitor, the Pal 5 globular cluster. We do not make use of measurements of the density along the stream.

To fit these data we follow the action-angle modeling approach of B14 (see also Sanders 2014). The approach of B14 consists of a simple analytic model for the frequency distribution of tidal debris at the time of stripping. In action-angle coordinates, this analytic model can be straightforwardly manipulated using the simple linear action-angle dynamics to derive the current frequency-angle structure along the stream for any given model. In particular, one can easily and analytically compute the track in frequency and angle space as a function of the location along the stream. Bovy (2014) also introduced a novel, general method for computing actions, frequencies, and angles in any static gravitational potential (including triaxial potentials) that can be used to convert this track in frequency-angle space to configuration space. The track can also be converted to configuration space in a more direct manner using the Torus Mapper code of Binney \& McMillan (2016), but we primarily use the method of B14, because we were unable to get the Torus Mapper to return action-angle coordinates that were as accurate as those obtained using the B14 method. We only use the Torus Mapper in the rare instances where the B14 method fails to converge. In configuration space, the track can be compared to observed data. The fundamental ingredients of the model are (a) a prescription for the times at which stars are stripped from the progenitor, (b) a model for the distribution of frequency (and angle) offsets from the progenitor at the time of stripping, (c) the phase-space location of the progenitor, and (d) the gravitational potential of the host galaxy (the Milky Way in this case). In the case of Pal 5, ingredient (c) is strongly constrained by the measurements of the position and velocity of the Pal 5 cluster. For GD-1, for which the progenitor is unknown, the phase-space location of the progenitor needs to be fully constrained by the stream data.

B14 proposed a simple model for ingredients (a) and (b), in which the stripping rate is constant up to a time $t_{d}$ in the past at which the disruption started and in which the frequency offset distribution is determined by a single velocity-dispersion parameter $\sigma_{v}$. B14 argued and demonstrated for a single $N$-body simulation of the disruption of a GD-1-like cluster that the stripping rate can be approximated as constant on timescales longer than the radial period; on shorter timescales the stripping is dominated by pericentric bursts. We have performed similar additional simulations in the three-component Milky-Way-like potential MWPotential2014 and for clusters that are more concentrated than the loosely-bound cluster used by B14 and find the same behavior in all cases. Furthermore, we have computed the effect on the track of assuming a stripping rate that decreases or increases in time. In all of these cases, the mean frequency offset between the stream and the progenitor changes by less than $5 \%$ or less than $1 / 3$ of the dispersion around the mean frequency. Thus, the track is largely insensitive to changes in the stripping rate and we assume that it is constant throughout the analysis.

B14 further demonstrated that the mean frequency vector along a stream is constant up to the edge of the stream, which can only be reached by stars stripped at exceptionally high frequency differences (their Figure 5). It is straightforward to compute the density along the stream in the B14 model in a similar manner as the calculation of the mean frequency along the stream and such calculations demonstrate that the edge of the stream has much lower density than the part closer to the progenitor. Thus, the segment of the stream that is high enough in surface brightness to be detected observationally has an approximately constant frequency vector. Therefore, we can model this part of the stream as a single orbital torus. Note that this does not mean that we can model it as a single orbit, because the direction of the frequency offset between the progenitor and the stream is in general not aligned with the direction of the frequency vector of an orbit. Therefore, the path along the torus along which the stream lies is not aligned with an orbit on the torus. This is especially the case in the three-component Milky Way models and the non-spherical halos that we consider 

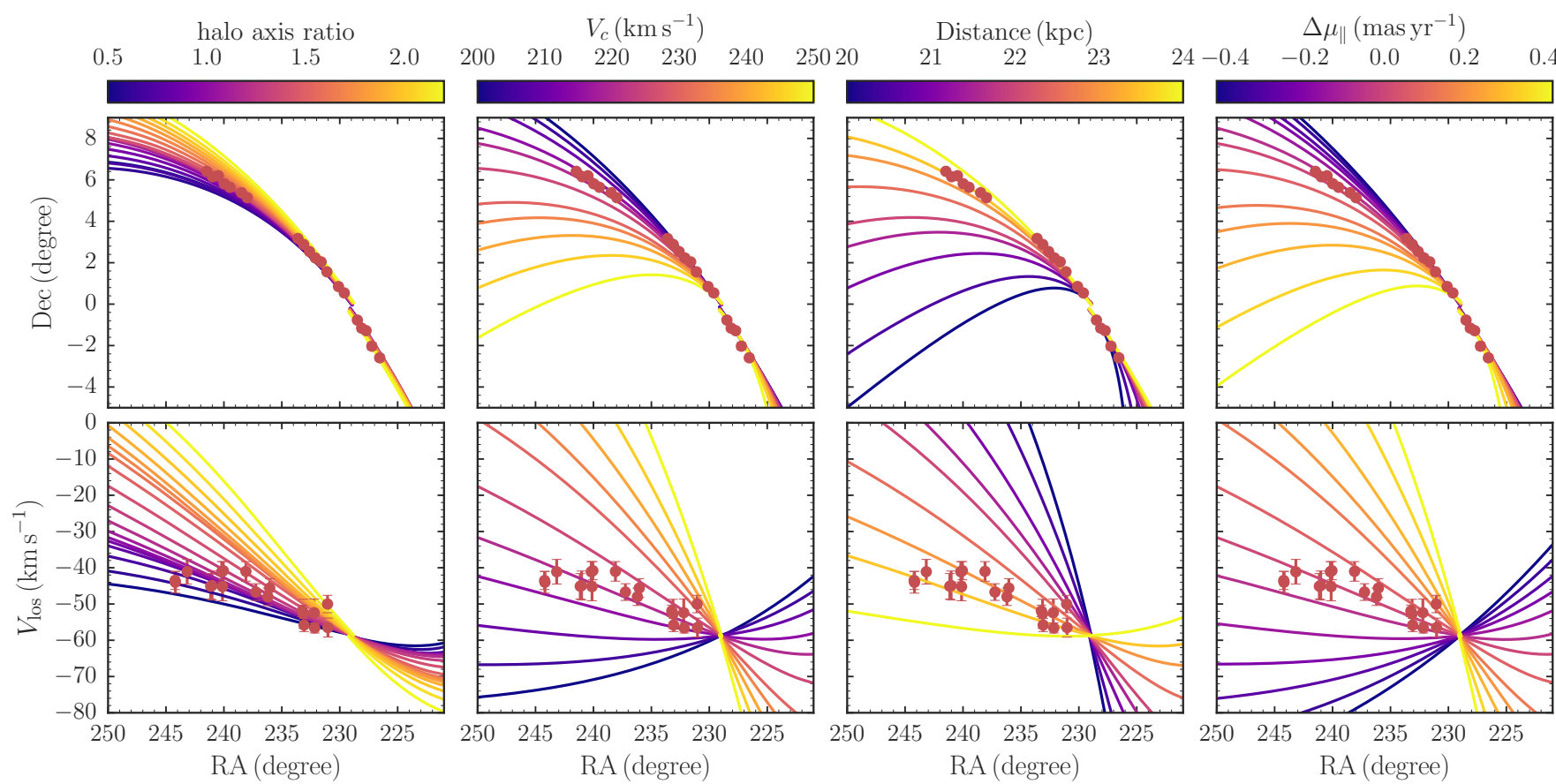

Fig. 2.- Sensitivity of the Pal 5 stream location to variations in the gravitational potential and in the position and proper motion of the Pal 5 progenitor cluster. The top panels display the location of the stream on the sky and the bottom panels give the line-of-sight velocity along the stream and include the data that we fit (see text). The leftmost column displays variations in the axis ratio $c / a$ of the halo, which changes the relative magnitude of the radial and vertical components of the force at Pal 5's location. The second column shows variations in the circular velocity $V_{c}$ at $R_{0}$, which changes the magnitude of the force, while keeping the ratio of the vertical-to-radial components the same. The third and fourth columns demonstrate the effect of changing the distance and the parallel component of the proper motion of the Pal 5 progenitor cluster. The overall normalization of the force (through $V_{c}$ ), the distance, and the proper motion all affect the location of the track in sky position and line-of-sight velocity in the opposite way (increasing Dec while decreasing the velocity at a given RA) and they are therefore to a large extent degenerate with each other. However, varying the axis ratio changes the track in both coordinates in the same direction and is therefore uncorrelated with the other parameters. The Pal 5 stream is exquisitely sensitive to the halo's axis ratio.

here. We do not know the extent of either the Pal 5 or GD-1 stellar streams because they are limited by the edge of the surveys in which they have been detected or by the Galactic plane. Therefore, constraining the time $t_{d}$ at which disruption started is difficult with the present data. We therefore fix $t_{d}$ to $10 \mathrm{Gyr}$ in both cases, making the stream old enough that for any potential that we consider a long stream forms. If better measurements of the width and length of streams were available, these could be used as additional constraints, because in very non-spherical halos it is difficult to produce a thin and long stream. However, for the halo shapes that we find to be consistent with the Pal 5 and GD-1 data below, this is not a major problem.

When we model the stream as being a single orbital torus, we can further restrict the model. The parameter $\sigma_{v}$ determines the location of the track, because it scales the frequency offset between the progenitor and the stream, and it sets the width and length of the stream (in conjunction with $t_{d}$ ). We do not attempt to match the observed width of the streams below and therefore $\sigma_{v}$ only affects the location of the track. That said, the $\sigma_{v}$ that produce a matching tracks below also give reasonable stream widths. For Pal 5 we fix the known sky position and line-of-sight velocity of the progenitor and we use the dispersion parameter $\sigma_{v}$ as a way to match the stream-progenitor offset. For GD-1, for which we do not know the progenitor position, $\sigma_{v}$ would be degenerate with the phase-space location of the progenitor and we therefore fix it to a reasonable value. Similarly, one of the six phase--space coordinates of the GD-1 progenitor is unconstrained by this modeling and we fix one of the sky location coordinates near one end of the GD-1 stream. Because we can approximate the stream track as having constant frequency, it also does not matter whether we model GD-1 as being a leading or trailing arm. This choice excludes the possibility that GD-1's progenitor or dissolved progenitor sits in the middle of the observed part of the stream. This is unlikely because in this case a clear kink of $\Delta \phi_{2} \approx 0.5^{\circ}$ would be visible at the $\phi_{1}$ position of the progenitor, which is not observed.

The Milky-Way potential model of $\S 2.1$ is characterized by 8 parameters and the stream model adds four (in the case of Pal 5) or five (for GD-1) additional parameters. To lower the dimensionality of each stream fit and thus speed it up, we restrict the range of potentials as follows. We perform the fit described in $\S 2.2$ fixing $R_{0}=8 \mathrm{kpc}$ and $V_{c}=220 \mathrm{~km} \mathrm{~s}^{-1}$ and we obtain 32 independent samples from the posterior PDF for the remaining six parameters. For each of these 32 samples we then analyze the stream data separately, keeping the five potential parameters describing the relative amplitudes, the scale length and height of the disk, and the scale radius of the halo fixed at the value of the sample, but allowing $c / a$ and additionally $V_{c}\left(R_{0}\right)$ to vary (between $1 / 2$ and 2 for $c / a$ and between $200 \mathrm{~km} \mathrm{~s}^{-1}$ and $250 \mathrm{~km} \mathrm{~s}^{-1}$ for $\left.V_{c}\left(R_{0}\right)\right)$. These 32 sampled sets of fixed parameters are shown as crosses in Figure 1 versus the $c / a$ of each sample, which is not held fixed. All of these are a priori reasonable poten- 

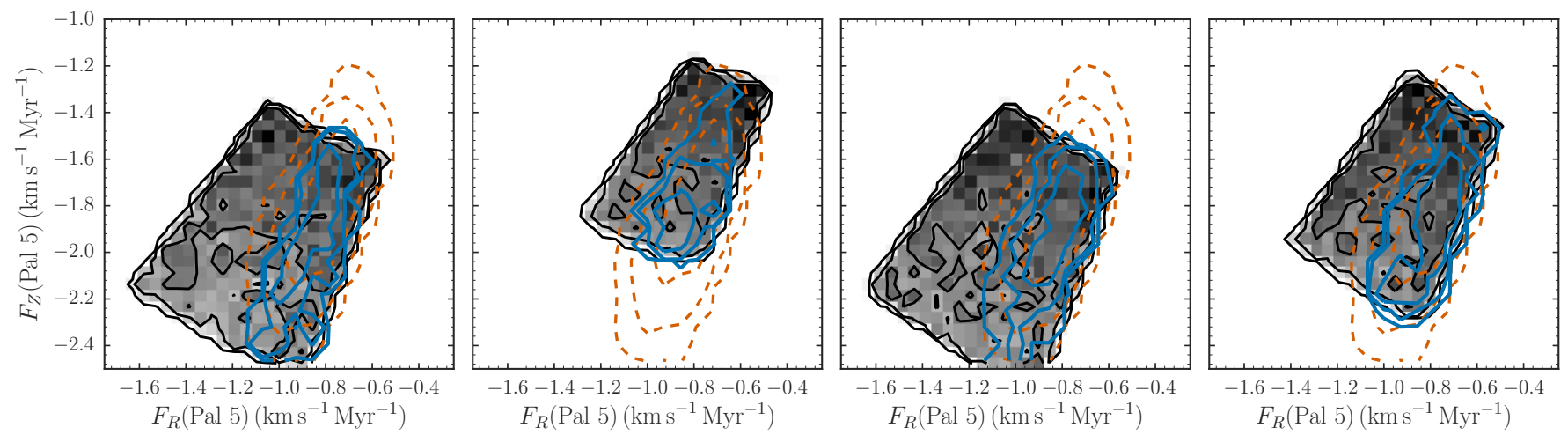

FIG. 3.- Example prior and posterior PDFs for the force at the fiducial location of the Pal 5 cluster for four of the 32 considered potential families. These four examples were chosen to illustrate the range of force fields included in the modeling. The grayscale density and black contours display the prior on the force field (from varying the halo axis ratio and circular velocity uniformly within their allowed range for each potential family). The solid blue curves show the posterior PDF for each potential family. The dashed orange curve displays the posterior PDF considering all potential families simultaneously. Each potential family agrees on the constraint on the radial and vertical components of the force at the location of Pal 5 (to the extent allowed by the prior), even though their prior ranges are very different.

tial models, as $c / a$ and $V_{c}\left(R_{0}\right)$ are almost uncorrelated with the other potential parameters (see Figure 1). Thus, for each of the 32 samples, we sample a wide range of halo shapes and overall Milky-Way masses and between the 32 families we sample a wide range of allowed disk and halo models. Combining these 32 independent Markov Chain Monte Carlo (MCMC) chains requires one in principle to compute the relative evidence or marginal likelihood to weight them, but we find that in each of the 32 potential families equally good fits to the stream can be obtained and we simply weight all of the chains equally. We will discuss this in more detail below, as it has important implications for what information is obtained about the gravitational potential from a single stream.

To transform between Galactocentric coordinates and heliocentric coordinates, we fix $R_{0}=8 \mathrm{kpc}$, the Sun's height above the mid-plane $Z_{0}=25 \mathrm{pc}$, and the Solar motion with respect to a circular orbit to $\left(V_{R, \odot}, V_{T, \odot}, V_{Z, \odot}\right)=(-11.1,24.0,7.25) \mathrm{km} \mathrm{s}^{-1}$ (Schönrich et al. 2010; Bovy 2015). Even a $\approx 10 \mathrm{~km} \mathrm{~s}^{-1}$ uncertainty in $V_{T, \odot}$ only affects the inferred force by $<10 \%$, which is smaller than the statistical uncertainties that we find below.

To summarize our stream-modeling methodology:

- We only use the observed location of the stream track, not the density along the track or its width or extent.

- We approximate each stream as a single orbital torus, assuming a single frequency-offset between the progenitor and every location along the observed part of the stream. We fix the time at which disruption started to $t_{d}=10 \mathrm{Gyr}$, because it does not affect the location of the track in the single-torus approximation and cannot be determined without an observation of the extent of the stream;

- We vary the unknown or marginally-constrained phasespace coordinates of the progenitor of the stream (the distance and proper-motion vector for Pal 5 and the sky location, distance, proper motion, and line-of-sight velocity for GD-1). For Pal 5 we vary the velocity-dispersion parameter $\sigma_{v}$, but we do not for GD-1, because it is degenerate with the unknown location of the progenitor.

- We consider a wide range of three-component potential models by sampling 32 plausible values of the relative disk-halo-bulge contribution and of the scale parameters of the disk and halo and by for each of these 32 families fully varying the halo axis ratio $c / a$ and the circular velocity $V_{c}\left(R_{0}\right)$ without any prior constraint.

\section{ANALYSIS OF PAL 5}

\subsection{Data and parameter sensitivity}

For Pal 5, the available data on the stream that we use are (a) the stream sky position measurements from SDSS from Fritz \& Kallivayalil $(2015)^{4}$ and (b) the line-ofsight velocity measurements of individual stream members from Kuzma et al. (2015). Because the latter do not resolve the velocity dispersion within the stream, we use the individual-member measurements as if they were measurements of the line-of-sight velocity of the mean stream track. We use the position measurements as the measured declination (Dec) of the track at a given right ascension (RA) plus its uncertainty and the line-of-sight velocities similarly as a function of RA. These data are shown in Figure 2. While we will show the sky location of the leading arm, we do not use it in the fit because it is so short, but takes about as long to predict as the trailing arm.

We further fix the following coordinates of the Pal 5 cluster as the stream progenitor: $\mathrm{RA}=229^{\circ} .018$, Dec $=-0.124$, and $V_{\mathrm{los}}=58.7 \mathrm{~km} \mathrm{~s}^{-1}$. While the lineof-sight velocity has $\mathrm{a} \approx 1 \mathrm{~km} \mathrm{~s}^{-1}$ uncertainty, this is much smaller than the uncertainty in the distance and proper motion of the Pal 5 cluster and it therefore does not affect the stream track significantly. We use the measurement of the proper motion of the Pal 5 cluster from Fritz \& Kallivayalil (2015): $\left(\mu_{\alpha} \cos \delta, \mu_{\delta}\right)=$ $(-2.296,-2.257) \pm(0.186,0.181)$ mas $\mathrm{yr}^{-1}$. However, the proper motion uncertainty is large enough that it has a big impact on the location of the stream track. We include the proper motion in our fits and add the Fritz \& Kallivayalil (2015) measurement to the likelihood. Because we find that the direction of the Fritz \& Kallivayalil (2015) proper-motion measurement is almost exactly aligned with the direction of the stream, we use an alternative parameterization of the proper motion in terms of

${ }^{4}$ We note that there is a typo in Table 1 of Fritz \& Kallivayalil (2015). The point at $($ RA,Dec $)=(229.11,0.54)$ should instead be $(229.61,0.54)$. The arXiv version of the paper has been corrected. 

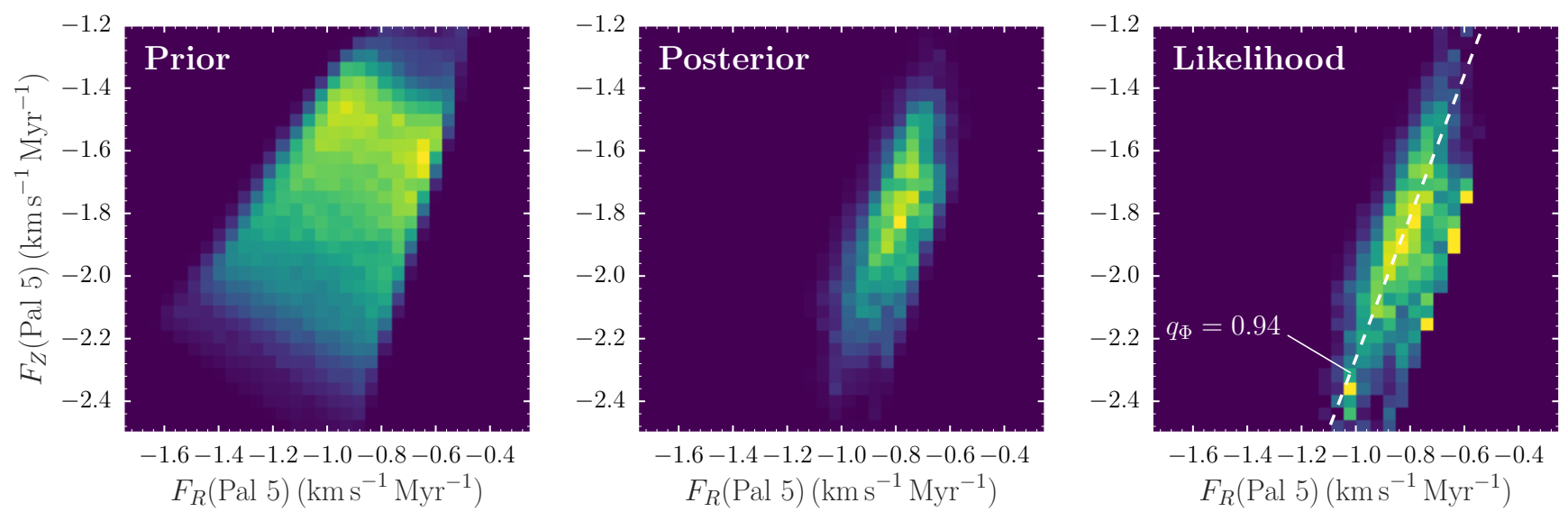

FIG. 4.- Constraint on the radial and vertical force components at the location of the Pal 5 cluster from the Pal 5 stream data. The left panel displays the prior on the force from all 32 potential families that we consider and the middle panel shows the posterior PDF combining all of the 32 independent potential-family MCMC chains. The right panel gives the ratio of the posterior and prior to demonstrate the measurement of the force coming from the Pal 5 stream data. The likelihood is narrowest perpendicular to the direction of constant overall potential flattening: The dashed curve shows the location of constant $q_{\Phi}=0.94$. A weaker constraint is obtained along the direction of constant flattening.

a component $\Delta \mu_{\|}$in the direction of $(2.296,2.257)$ and a component $\Delta \mu_{\perp}$ perpendicular to this direction (toward $(2.257,-2.296))$. Measurements of the distance to the Pal 5 cluster range from $20.9 \mathrm{kpc}$ to $23.2 \mathrm{kpc}$ (Harris 1996; Vivas \& Zinn 2006; Dotter et al. 2011) and we conservatively assume a flat prior on the distance between $20 \mathrm{kpc}$ and $24 \mathrm{kpc}$.

To get a sense of how the various model parameters affect the location of the Pal 5 stream, we have computed the stream track varying the halo axis ratio, the circular velocity, and the distance and parallel propermotion offset. As the fiducial model around which these parameters are varied we assume a distance of $23.2 \mathrm{kpc}$, the Fritz \& Kallivayalil (2015) best-fit proper motion, a stream velocity-dispersion parameters $\sigma_{v}=0.2 \mathrm{~km} \mathrm{~s}^{-1}$, and the best-fit three-component potential to the data in $\S 2.2$ obtained by fixing $c / a=1, V_{c}\left(R_{0}\right)=220 \mathrm{~km} \mathrm{~s}^{-1}$, and $R_{0}=8 \mathrm{kpc}$ (this potential is almost exactly the same as MWPotential2014, except that its parameters have not been rounded to convenient values; see Bovy 2015). These track variations are displayed in Figure 2. It is clear that the track, both on the sky and in line-ofsight velocity, varies significantly when the parameters are varied over a reasonable range. In particular, the circular-velocity, the distance, and the proper motion of the progenitor affect the stream track in a similar manner in both sky position and velocity. Therefore, in fitting these data a model has significant leeway to trade differences in $V_{c}\left(R_{0}\right)$ for differences in the distance and proper motion of the progenitor.

However, it is also immediately clear from Figure 2 that changing the halo axis ratio, which changes the overall flattening of the potential and thus the ratio of the vertical and radial components of the force, changes the sky position and the line-of-sight velocity of the stream track in a manner that is perpendicular to changes induced by the other model parameters. That is, when $V_{c}$ is increased, the distance is decreased, or the proper motion is increased, the trailing tail of the stream moves toward higher declinations and lower velocities. When the halo axis ratio is increased, the trailing track moves toward both higher declinations and higher velocities. Thus, when fitting the data, the halo axis ratio can be measured independently from the other parameters, which among themselves will have a high degree of degeneracy.

\subsection{Pal 5 Potential constraints}

We explore the posterior PDF of each of the 32 potential families (see above) using MCMC and combine all chains to provide a sampling of the model parameters. We find the halo axis ratio to be $c / a=0.9 \pm 0.2$. As expected, we find that the halo axis ratio is uncorrelated with $V_{c}$ or the distance and parallel proper-motion offset of the progenitor. There is a slight degeneracy between $c / a$ and the component of the proper motion perpendicular to the Fritz \& Kallivayalil (2015) measurement, but the perpendicular component itself is found to be well constrained $\left(\Delta \mu_{\perp}=0.04 \pm 0.03\right.$ mas yr $\left.^{-1}\right)$ so this only has a small impact and a much better proper-motion measurement would be necessary to reduce the degeneracy. That our constraint on $\Delta \mu_{\perp}$ is so close to zero is surprising given the $\approx 0.2$ mas yr $^{-1}$ uncertainty in its direct measurement by Fritz \& Kallivayalil (2015), but this is simply due to chance. The parameters $V_{c}$, the distance $D_{\mathrm{Pal}} 5$, and parallel proper-motion offset are mutually degenerate: we find $V_{c}\left(R_{0}\right)=225 \pm 14 \mathrm{~km} \mathrm{~s}^{-1}$, $D_{\text {Pal } 5}=22.9_{-0.9}^{+0.6} \mathrm{kpc}$ (the asymmetric uncertainty is due to our prior that $\left.D_{\mathrm{Pal} 5}<24 \mathrm{kpc}\right)$, and $\Delta \mu_{\|}=$

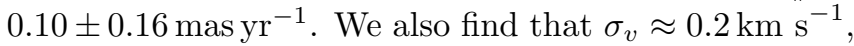
with only a minor correlation with $\Delta \mu_{\perp}$. This $\sigma_{v}$ leads to a stream width of $\approx 20^{\prime} \mathrm{FWHM}$, in good agreement with the measurement from Carlberg et al. (2012), especially considering that we assume an age of $10 \mathrm{Gyr}$ for the Pal 5 stream. This $\sigma_{v}$ also gives a line-of-sight velocity dispersion in the stream of $\approx 1 \mathrm{~km} \mathrm{~s}^{-1}$, at the low end of, but consistent with the measurements from Odenkirchen et al. (2009) and Kuzma et al. (2015), which give a velocity dispersion of $\approx 2 \mathrm{~km} \mathrm{~s}^{-1}$.

As already mentioned in $\S 3$ above, we find that each of the 32 potential families essentially fits the stream equally well (a single potential did not converge in a short enough time and is not included in the following analysis, although it does not change the results). These poten- 

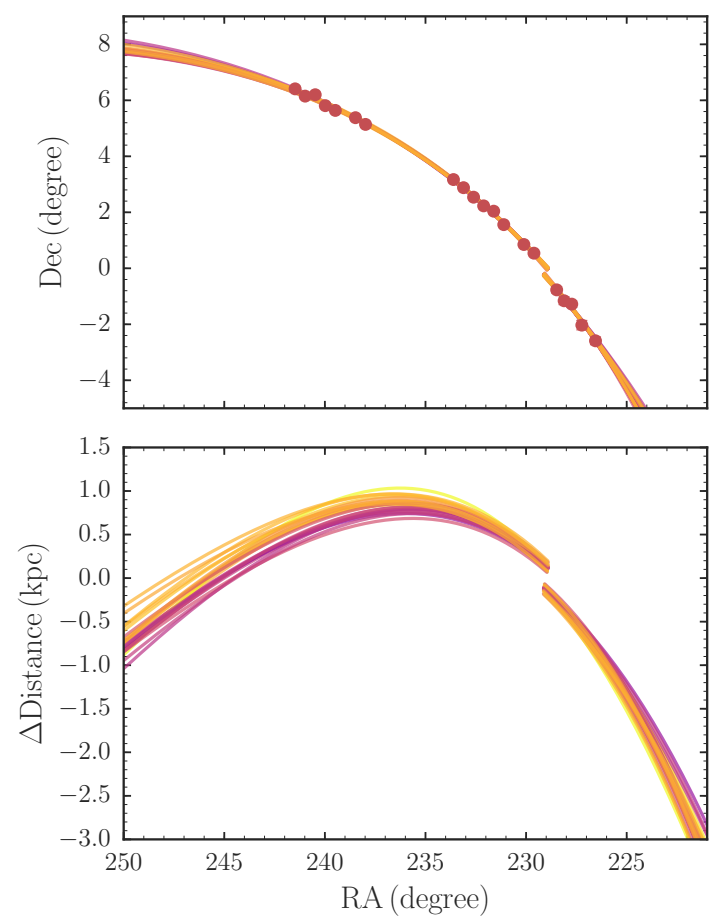

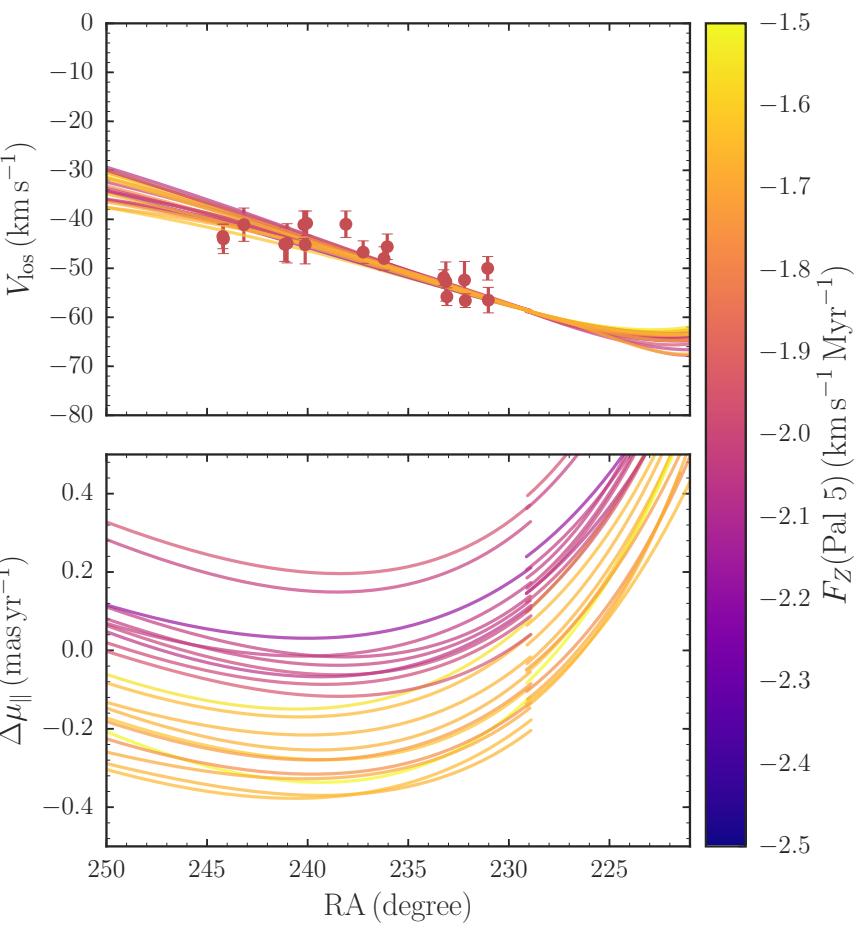

FIG. 5.- Posterior samples from the fit to the Pal 5 stream data. The samples are color-coded by their value of the vertical component of the force at Pal 5 to illustrate how the measurements of the individual vertical and radial components of the force can be improved by future data. All of the displayed models fit the data well; the leading arm's sky location was not included in the fit, but is successfully recovered. Measurements of the sky location or radial velocity of the trailing arm at higher RA do not distinguish well between different forces. All models have approximately the same distance gradient along the stream. Better measurements of the proper motion of the Pal 5 cluster or stream would allow much improved measurements of the force field near Pal 5.

tials explore a wide range of disk-to-halo ratios, disk scale lengths, and halo concentrations. Therefore, it is clear that the observed track of the stream does not hold much information about the global properties of the gravitational potential. An arm of a stream is approximately at a constant frequency and therefore corresponds to a single orbital torus along which energy should be conserved. This energy conservation is ensured by balancing the work done by the kinetic energy when going from one end of the stream to the other. Thus, we expect the force along the stream to be a robustly measured quantity. To test this hypothesis, we compute the force in all of the potentials that we consider at a fiducial location for the Pal 5 cluster. That is, we fix the distance to be $23.46 \mathrm{kpc}$, which places the cluster at $(R, Z)=(8.4,16.8) \mathrm{kpc}$; this conveniently has $Z / R=2$.

Examples of prior and posterior PDFs for the radial and vertical components of the force at the fiducial Pal 5 location are displayed in Figure 3 for four of the 32 potential families that we consider (chosen to illustrate the range of force fields included in our models). The grayscale density shows the prior on the radial and vertical components of the force when varying the parameters of the potential model, $c / a$ and $V_{c}$ for each potential: curves of constant $c / a$ run from the upper-right to the lower-left edge, curves of constant $V_{c}$ run from the upperleft to the lower-right edge. The blue contours display the posterior PDF for the particular potential and the orange contours are the posterior PDF from considering all of the potentials. This figure demonstrates that even though the prior on the force can vary significantly among the 32 potential families, the posterior PDF always prefers the same force (as much as allowed by each particular potential's prior on the force).

The prior, posterior, and their ratio - the likelihoodconsidering all potentials is shown in Figure 4. The prior is obtained by sampling $c / a$ and $V_{c}$ from their prior range for all 32 potential families and computing the radial and vertical components of the force for all of these potentials. The middle panel displays the posterior PDF, which is much narrower than the prior. Because even the uninformative, flat priors on $c / a$ and $V_{c}$ lead to a non-flat prior for the force (the left panel), we need to divide the posterior by the prior in the two-dimensional plane of the force components to know what information about the force is obtained from the Pal 5 data alone. This is shown in the left panel. The likelihood is similar to the posterior, but slightly wider. This anisotropy in the likelihood is expected from Figure 2: the flattening is uncorrelated with the other model parameters, while the normalization of the force is correlated with the distance and proper motion of the Pal 5 cluster.

The likelihood in Figure 4 displays a clear degeneracy between the radial and vertical components of the force. The dashed line shows the location of a constant flattening of the total potential, defined as

$$
q_{\Phi}^{2}=\frac{Z}{R} \frac{F_{R}}{F_{Z}},
$$

where $Z / R=2$ for Pal 5 . It is clear that the degeneracy between $F_{R}$ and $F_{Z}$ runs along the line of constant $q_{\Phi}$. The constraint in the direction perpendicular to this line 

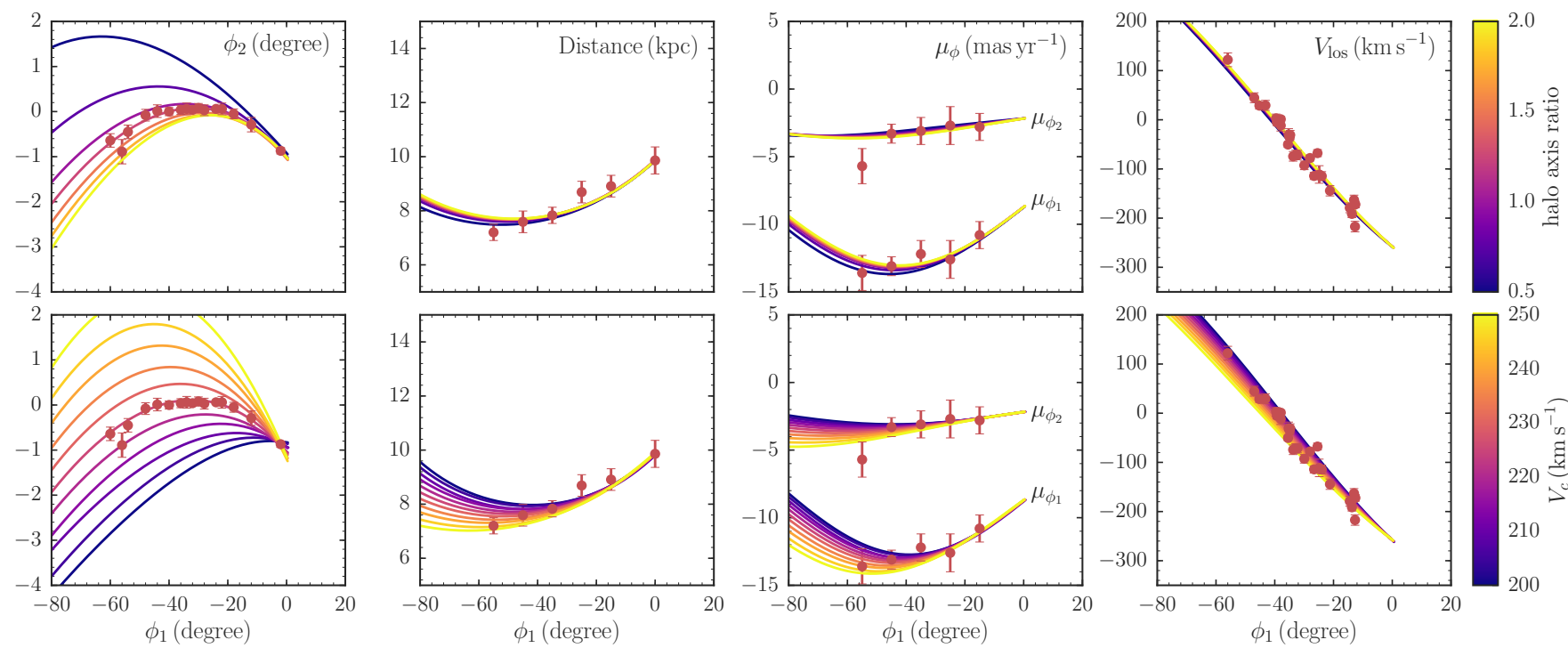

FIG. 6. - Sensitivity of the GD-1 stream track to variations in the gravitational potential. The top panels demonstrate the effect of changing the halo axis ratio, which effectively changes the ratio of the vertical to the radial components of the force near the GD-1 stream. The bottom panels show the effect of varying $V_{c}\left(R_{0}\right)$, which changes the magnitudes of the total force, while keeping their ratio the same. Only the stream's location on the sky (left panels) is sensitive to the halo axis ratio $c / a$; the distance or velocity components are hardly affected by changing $c / a$. All phase-space coordinates of the stream track are sensitive to $V_{c}$. Thus, the distance and velocity measurements constrain the location of GD-1's progenitor and $V_{c}$, while the sky location allows the halo axis ratio to be measured.

is three times narrower than that parallel to the line.

We can therefore summarize the information about the Milky Way's gravitational potential learned from the Pal 5 stream data in the direction of the two principal directions of the likelihood in Figure 4 in the following convenient manner:

$$
q_{\Phi}=0.94 \pm 0.05
$$

and

$$
0.94^{2}\left(F_{R}+0.80\right)+2\left(F_{Z}+1.82\right)=-0.2 \pm 0.6
$$

where $F_{R}$ and $F_{Z}$ are measured in $\mathrm{km} \mathrm{s}^{-1} \mathrm{Myr}^{-1}$ at $(R, Z)=(8.4,16.8) \mathrm{kpc}$ and $\phi=178^{\circ} .4$.

Samples from the MCMC chains are compared with the data in Figure 5. All of these fit the data well. As discussed above, we did not include the sky location of the leading arm in the fit, but it is nevertheless well recovered. We also show the predicted track of the stream in distance and (parallel) proper motion. The weak distance gradient is consistent with the weak gradient observed by Ibata et al. (2016). The samples are colorcoded by the value of the vertical force component at Pal 5 's location, to illustrate how the measurement in equation (5) could be improved by future data. It is clear that better measurements of the sky location or line-of-sight velocity, even at larger distances from the cluster, do not distinguish between different values of $F_{Z}$ well. Similarly, measurements of the distance gradient along the stream will not improve the force measurements, because the gradient is similar in all allowed models. However, a better measurement of the proper motion of the Pal 5 cluster or a measurement of the stream's proper motion (both with $\sigma_{\mu} \lesssim 0.1$ mas yr$^{-1}$ ) would significantly improve the force measurements.

\subsection{Comparison to Küpper et al.}

A previous analysis of similar Pal 5 stream data was performed by Küpper et al. (2015), who claim tight constraints on the mass, scale radius, and potentialflattening $q_{\Phi}^{h}$ of the dark-matter halo based on these data. We find, however, that the Pal 5 stream data on their own contain very little information about the mass and scale radius of the dark-matter halo. To investigate why our results differ from those of Küpper et al. (2015), we perform some simple experiments. First, we sample the parameters of the potential model used by Küpper et al. (2015) using the same flat priors as Küpper et al. (2015), but requiring that $V_{c}\left(R_{0}\right)$ is between $200 \mathrm{~km} \mathrm{~s}^{-1}$ and $280 \mathrm{~km} \mathrm{~s}^{-1}$ (their prior on $V_{c}\left(R_{0}\right)$ ) and that the overall potential flattening is constrained to be $q_{\Phi}=0.94 \pm 0.05$, as we find from the Pal 5 data. We find no preference for any mass or scale radius of the dark-matter halo in this case, except for a strong degeneracy between these quantities because of the limited range in $V_{c}$. However, we find that $q_{\Phi}^{h}=0.95 \pm 0.15$ in good agreement with Küpper et al. (2015). We can add in a constraint similar to that found in equation (5), but changed to be consistent with Küpper et al. (2015)'s best-fit total force amplitude (which is consistent with ours within the uncertainties). In this case, we still find that there is hardly any constraint on the mass or scale radius of the halo, except that the allowed mass range for a given scale radius is reduced; the constraint on $q_{\Phi}^{h}$ remains the same.

Thus, we conclude that our measurements in equations (4) and (5) are weaker than those claimed by Küpper et al. (2015). This difference may be due to the fact that Küpper et al. (2015) ascribe and fit overdensities along the stream as resulting from pericentric stripping episodes, thus in essence adding a constraint on the radial frequency of the orbit. Because the significance of the observed overdensities is contested (Thomas et al. 2016) and because overdensities could be due to subhalo encounters (e.g., Carlberg et al. 2012; Bovy et al. 2016), not employing the overdensities as a constraint on the gravitational potential appears to be the conser- 


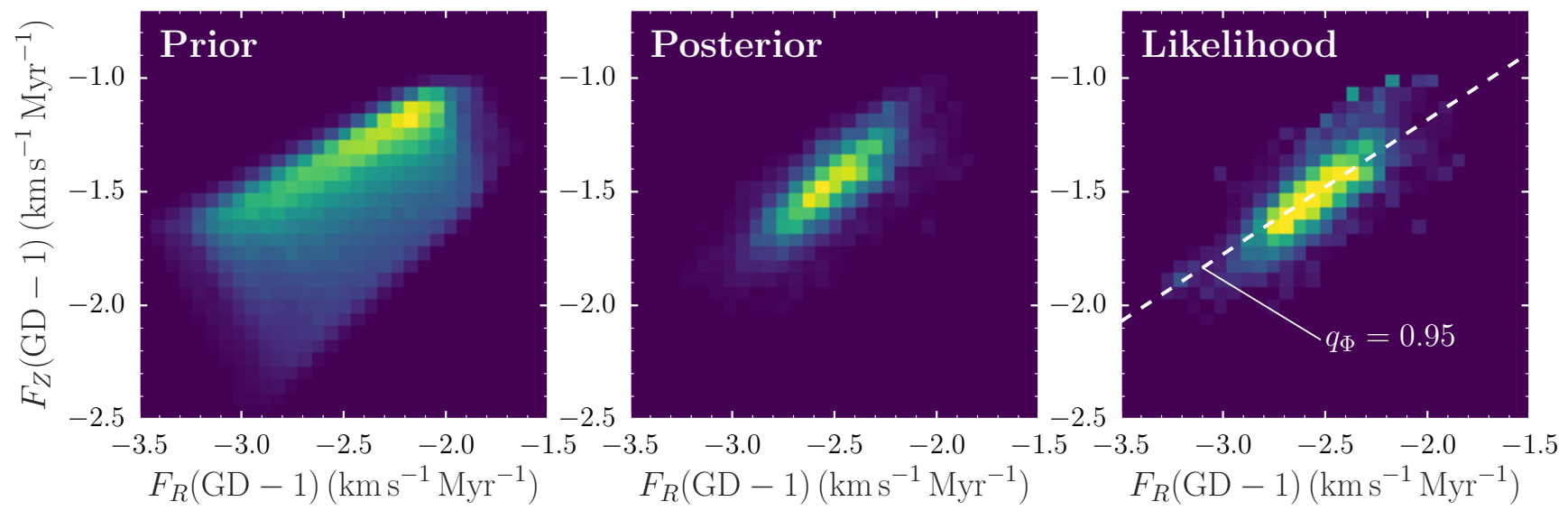

Fig. 7.- Like Figure 4, but for the GD-1 analysis. As for Pal 5, the likelihood is narrowest perpendicular to the direction of constant overall potential flattening. The dashed curve displays the location of $q_{\Phi}=0.95$, the best-fit potential flattening. The GD-1 data also provide a good constraint on the component of the force along the direction of constant flattening.

vative thing to do. In summary, our measurements of $q_{\Phi}$ and the force at Pal 5 are consistent with, but more conservative than the measurements of Küpper et al. (2015).

\section{ANALYSIS OF GD-1}

\subsection{Data and parameter sensitivity}

For GD-1, we fit the data from Koposov et al. (2010), which is shown in Figure 6. These data come in the form of the location of the track in a set of custom sky coordinates $\left(\phi_{1}, \phi_{2}\right)$ with $\phi_{1}$ approximately aligned with the stream. We use the measurements of the stream phasespace location as a function of $\phi_{1}$ in $\phi_{2}$, distance, proper motion, and line-of-sight velocity. Similar to the analysis of the Pal 5 data above, we also treat the line-of-sight velocity measurements of stream members as measurements of the track location in line-of-sight velocity at each star's $\phi_{1}$, because these measurements do not resolve the velocity dispersion of the stream. Because for GD-1 we have data on all six phase-space coordinates of the stream itself, the location of the progenitor - in our approximation of the stream track - is much better constrained than that of Pal 5 (especially because the distance to GD-1 is much better constrained than that to Pal 5 above). As discussed above, we fix the progenitor's location to $\phi_{1}=0^{\circ}$ and $\sigma_{v}=0.4 \mathrm{~km} \mathrm{~s}^{-1}$ and thus model the GD-1 stream as being a leading arm. We stress that this is simply the location of the progenitor in our approximation, not the true location of the progenitor.

We explore the sensitivity of the stream track to the gravitational potential in Figure 6. Similar to the sensitivity analysis for Pal 5 in Figure 2, we use the bulge, disk, and halo parameters of the best-fit three-component potential to the data in $\S 2.2$ obtained by fixing $c / a=1$, $V_{c}\left(R_{0}\right)=220 \mathrm{~km} \mathrm{~s}^{-1}$, and $R_{0}=8 \mathrm{kpc}$ as the fiducial potential family, but we find the best-fitting $c / a, V_{c}\left(R_{0}\right)$, and GD-1 progenitor parameters by fitting to the GD-1 data in order to have a fiducial model that fits the data well for this sensitivity analysis. We then vary $c / a$ and $V_{c}$ around their best-fitting values and compute the stream track in all phase-space components. The results from this are displayed in Figure 6.

It is clear from Figure 6 that varying the halo axis ratio has almost no effect on the stream's track in dis- tance or any of the velocity components. Changing $V_{c}$ has a small effect in the same components. However, the stream's location on the sky varies significantly for different $c / a$ or $V_{c}$. Within this potential family, $V_{c}$ can be constrained by the stream's distance, proper motion, and line-of-sight velocity independent of $c / a$ and the stream's location in the sky can then essentially constrain $c / a$. Thus, we again expect little to no correlation between the constraints on $c / a$ and those on the other parameters of the model for the GD-1 data. $V_{c}$ will similarly again be somewhat degenerate with the phase-space location of the progenitor.

\subsection{GD-1 Potential constraints}

We explore the posterior PDF for the potential and progenitor parameters for GD-1 using MCMC for the 32 potential families. Because for GD-1, unlike for Pal 5 , we have measurements of all six phase-space components the fit is more strongly constrained and every potential family prefers similar parameters. We find that $c / a=1.27_{-0.22}^{+0.27}$ with no significant correlations with any of the other model parameters. The circular velocity is constrained to be $V_{c}\left(R_{0}\right)=225 \pm 10 \mathrm{~km} \mathrm{~s}^{-1}$, correlated with the proper motion of the progenitor. The progenitor is at $\left(\phi_{1}, \phi_{2}, D, \mu_{\phi_{1}}, \mu_{\phi_{2}}, V_{\mathrm{los}}\right)=\left(0^{\circ},-0.82 \pm 0.08,10.1 \pm\right.$ $0.2 \mathrm{kpc}, 0.0 \pm 0.3$ mas yr $^{-1},-0.15 \pm 0.10$ mas yr $^{-1},-257 \pm$ $5 \mathrm{~km} \mathrm{~s}^{-1}$ ). The only significant correlations between the progenitor parameters are between the proper motion components.

Similar to the fit to Pal 5 above, we find that each of the 32 potential families provides an equally-good fit to the GD-1 data. Thus, the GD-1 data again do not hold much information about the global properties of the gravitational potential. A similar investigation to that shown in Figure 3 demonstrates again that while the effective prior on the local radial and vertical force components differs significantly between the 32 potential families, the posterior PDF of the radial and vertical force components near GD-1 is almost exactly the same in all 32 cases. Here, we compute the force at $(R, Z)=(12.5,6.675) \mathrm{kpc}$ and $\phi=0^{\circ}$, chosen to be close to the center of the GD-1 stream data.

The prior and posterior PDF for the radial and vertical force components at the center of the GD-1 data is 

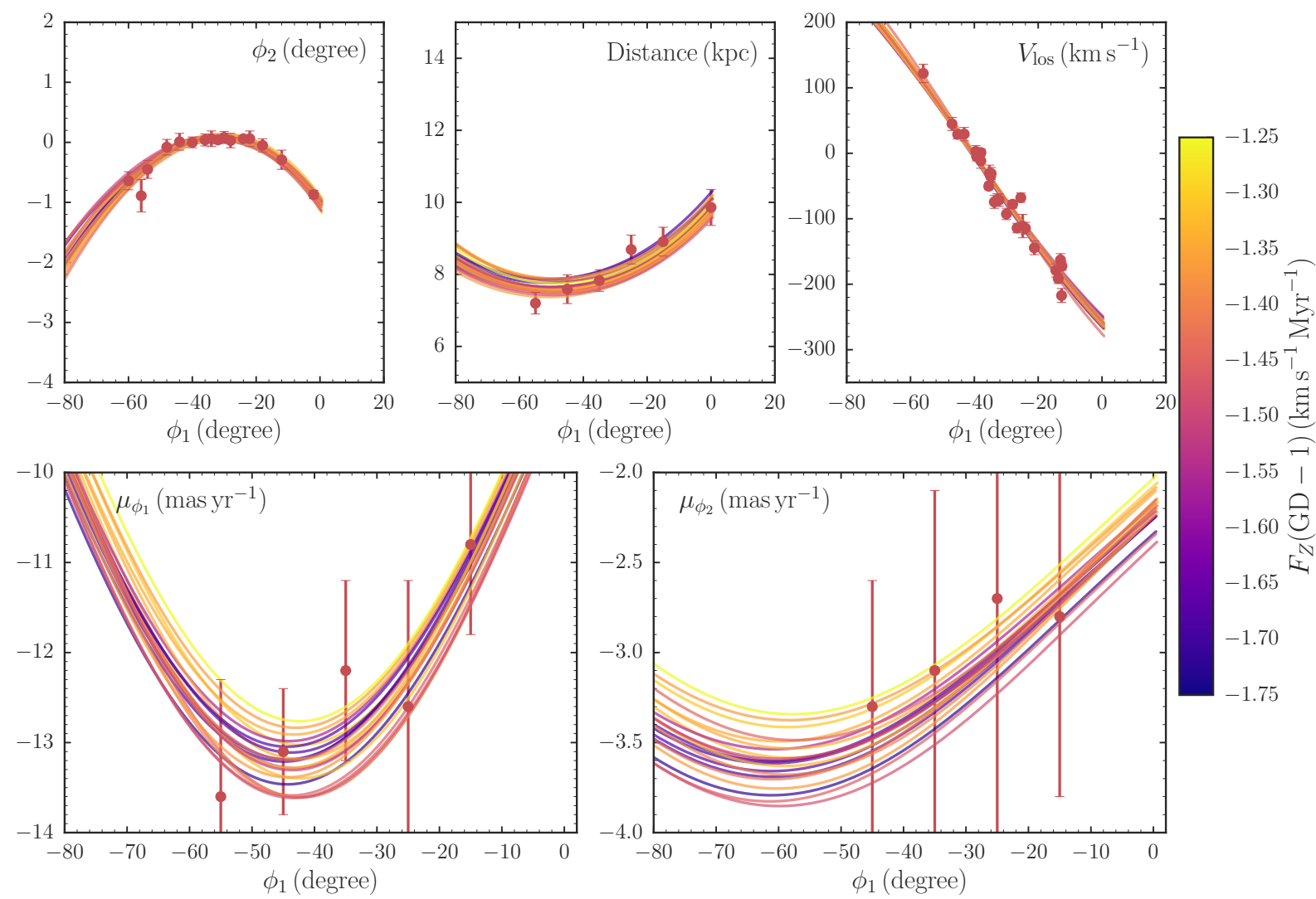

Fig. 8.- Posterior samples from the GD-1 stream fit. As in Figure 5, the samples are color-coded by their value of the vertical force component at GD-1 to illustrate how the measurements of the individual vertical and radial components of the force can be improved by future data. All of the displayed models fit the six-dimensional phase-space data for GD-1 well. Better measurements of the sky location, distance, or line-of-sight do not distinguish well between different forces. Improved, sub mas $\mathrm{yr}^{-1}$ measurements of the proper motion along the GD-1 stream would allow much improved measurements of the force field near GD-1.

displayed in Figure 7 combining the results from all 32 potential families. It is clear that the posterior is much more sharply peaked than the prior and that the GD-1 data therefore strongly constrain the force field in their vicinity. The ratio of the posterior and the prior (the likelihood) is shown in the right panel. Much as for Pal 5, the likelihood is anisotropic with the principal axes approximately aligned with the directions of constant overall potential flattening $q_{\Phi}$ and the direction perpendicular to this. For GD-1, the likelihood peaks at $q_{\Phi}=0.95$. The ratio of the widths in the two principal directions is again about three.

We can thus summarize the information about the gravitational potential provided by the GD-1 data alone as

$$
q_{\Phi}=0.95 \pm 0.04
$$

and

$0.95^{2}\left(F_{R}+2.51\right)+6.675 / 12.5\left(F_{Z}+1.47\right)=-0.05 \pm 0.3$

where $F_{R}$ and $F_{Z}$ are measured in $\mathrm{km} \mathrm{s}^{-1} \mathrm{Myr}^{-1}$ at $(R, Z)=(12.5,6.675) \mathrm{kpc}$ and $\phi=0^{\circ}$.

Samples from the MCMC chains for GD-1 are compared with the data in Figure 8 . All of these match the data well. The samples are color-coded by their value of the vertical force component near the center of the stream, to illustrate how the measurement in equation (7) could be improved by future data. It is clear that the main improvement would again come from sub mas $\mathrm{yr}^{-1}$ measurements of the proper motion along the stream.

\subsection{Comparison to Koposov et al. and Bowden et al.}

The GD-1 data that we fit in the previous subsection were previously analyzed by Koposov et al. (2010) and Bowden et al. (2015). Because Koposov et al. (2010) fit an orbit to these data, which is improper and has been superseded by the analysis of Bowden et al. (2015), we focus on the latter, but briefly return to Koposov et al. (2010) at the end of this subsection. Bowden et al. (2015) perform a similar fit as the one in this paper, fitting a stream track to the phase-space location of the data, but they compute the model stream tracks using orbit integration of tracer particles stripped from a model progenitor and they assume a simple flattened logarithmic potential rather than the three-component potential that we use. Apart from the difference in potential, this is essentially the same approach as we follow here, except that their prescription for the phase-space distribution of the tidal debris at the time of stripping is different from ours in detail and that we use action-angle coordinates to compute a smooth representation of the present-day track, while they use Monte-Carlo orbit integration to reconstruct the present-day track. Thus, we expect to 

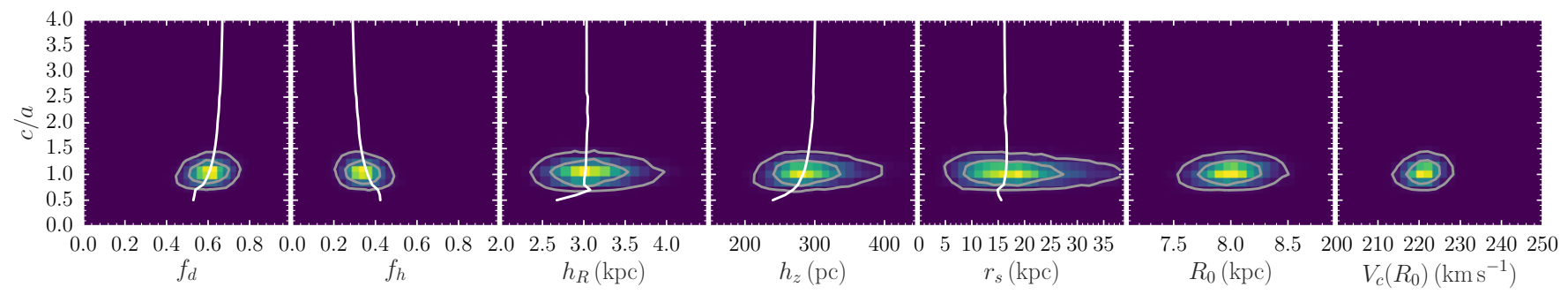

FIG. 9.- Constraints on the halo-axis ratio $c / a$ and the disk, bulge, and halo components of the Milky Way after adding the constraints derived from the Pal 5 and GD-1 stream to the data in $\S 2.2$. Compared to the weak constraint on $c / a$ from prior dynamical data in Figure 1, the Pal 5 and GD-1 data provide a good measurement of $c / a=1.05 \pm 0.14$ with no correlation with the other properties of the potential.

find essentially the same results as Bowden et al. (2015). However, our constraints on the potential flattening and $V_{c}$ are significantly tighter than those found by Bowden et al. (2015).

To investigate the cause of this difference, we have repeated our fit to the GD-1 data, but using a flattened logarithmic potential with free parameters $q_{\Phi}$ and $V_{c}$ $\left(\Phi=V_{c}^{2} / 2 \ln \left[R^{2}+z^{2} / q_{\Phi}^{2}\right]\right)$, setting $R_{0}=8.5 \mathrm{kpc}$ (but we get similar results for $R_{0}=8 \mathrm{kpc}$ ), and using the same flat priors as Bowden et al. (2015). In this case the fit is much faster than for our three-component potential families, because orbit integration in this simple potential is much faster, and we can obtain 10,000 MCMC samples in a matter of hours. We find that $q_{\Phi}=0.90 \pm 0.06$ and $V_{c}=212 \pm 15 \mathrm{~km} \mathrm{~s}^{-1}$ in this case. The constraint on $q_{\Phi}$ is weaker than in the case of the three-component potential models and is in good agreement with that of Bowden et al. (2015); we also recover the same correlation between $q_{\Phi}$ and $V_{c}$. Our best-fit value for $V_{c}$ is somewhat smaller than that of Bowden et al. (2015) although within the formal uncertainties, which may be due in part because of our higher assumed solar-motion component $V_{T, \odot . ;}$ the uncertainty in $V_{c}$ is similar. Overall, the two analyses agree well.

Therefore, we conclude that our modeling of the GD-1 data in terms of three-component potential families provides tighter constraints on the gravitational potential than using a simple logarithmic potential. This is probably due, at least in part, to the fact that the simple logarithmic potential does not realistically describe the structure of the potential. As shown by Williams et al. (2014), the offset between a tidal stream and a single orbit is very small for the flattened logarithmic potential and Bowden et al. (2015) also find that simply fitting the GD-1 data as an orbit returns the same results as their stream fit. However, GD-1 is close enough to the disk that in a more realistic potential the stream-orbit offset is larger and varies with $q_{\Phi}$ (Sanders \& Binney 2013). Comparing orbits to the stream tracks for Pal 5 and GD1 shown in Figures 2 and 6 directly demonstrates that the location of the tracks varies more than that of orbits when changing the potential. Provided that one is willing to assume that the potential is closer to a disk-plus-halo than to a logarithmic potential, this effect should lead to stronger constraints on the potential and our modeling of the potential in terms of three-component potential families capitalizes on this.

Finally, Koposov et al. (2010) claim that the GD-1 data cannot constrain the flattening of the halo without informative priors on the disk and only find a lower limit
$q_{\Phi}^{h} \gtrsim 0.9$ from the GD-1 data. We, however, find that the GD-1 data do provide an informative constraint on the shape of the halo. This is in part due to the fact that we only allow reasonable values for the mass of the disk. Even though our potential families explore a wide range of possible disk parameters, the disk always has $M_{\text {disk }} \lesssim 10^{11} \mathrm{M}_{\odot}$, while Koposov et al. (2010) allow unrealistic values above this value and have a maximumlikelihood $M_{\text {disk }} \approx 1.4 \times 10^{11} \mathrm{M}_{\odot}$. However, this is not the only cause of our better constraint, because we find the same best-fit value of $c / a \approx 1.25$ for all potential families and furthermore, $c / a$ is uncorrelated with $V_{c}\left(R_{0}\right)$, which scales the mass of the disk within each family. Thus, the main improvement comes from properly modeling the GD-1 data as a stream in a three-component potential, which leads to tighter constraints on the potential and on $c / a$ in particular. It is clear that the Pal 5 data provide a more direct measurement of the shape of the halo than the GD-1 data, because the Pal 5 data are located much further from the disk than the GD-1 data.

\section{COMBINED CONSTRAINTS ON THE SHAPE OF THE INNER DARK-MATTER HALO}

In sections 4 and 5 above, we inferred values of the halo axis ratio of $c / a=0.9 \pm 0.2$ and $c / a=1.27_{-0.22}^{+0.27}$ for the Pal 5 and GD-1 streams. These measurements were obtained by fitting general potential families, inspired by fits to data on the rotation curve, vertical kinematics, etc. from $\S 2.2$, but not required to provide a good fit to those data. We saw that each of 32 potential families provided an equally good fit to the stream data and that the inferred value of $c / a$ was uncorrelated with $V_{c}$, indicating that these $c / a$ values should not be significantly changed if we further require the potential to fit the constraints from $\S 2.2$. In this section we perform fits to the data in $\S 2.2$ plus the measurements for Pal 5 from equations (4) and (5) and for GD-1 from equations (6) and $(7)$.

First, we add the Pal 5 and GD-1 constraints separately, obtaining $c / a=0.93 \pm 0.16$ for Pal 5 and $c / a=1.3_{-0.3}^{+0.5}$ for GD-1, in line with the measurements from the stream data directly, but with slightly different uncertainties. The uncertainties for GD-1 are somewhat larger than those found from considering the 32 potential families in $\S 5.2$ because we no longer demand that $c / a<2$ - the prior in the analysis in $\S 5.2$ - and because we only use the GD-1 constraints on the force components, not the full GD-1 data. The $c / a$ values obtained for Pal 5 and GD-1 are uncorrelated with any of the other 
potential parameters describing the bulge, disk, and halo or with $R_{0}$ and $V_{c}\left(R_{0}\right)$. Because GD-1 is located at a Galactocentric radius of $\approx 14 \mathrm{kpc}$ and $\mathrm{Pal} 5$ at a radius of $\approx 19 \mathrm{kpc}$, this hints at a decrease in the axis ratio with Galactocentric radius, although the values are entirely consistent with each other.

Fitting all constraints simultaneously, we find that $c / a=1.05 \pm 0.14$, again with no correlation with any of the other parameters describing the potential. The $2 \sigma$ range spans $c / a=0.79$ to $c / a=1.33$. Figure 9 displays the joint two-dimensional posterior $\mathrm{PDF}$ for $c / a$ versus all of the other model parameters, similar to Figure 1 before the addition of the stream data. The halo is thus constrained to be spherical to about $15 \%$. The scale radius of the dark-matter halo is constrained to be $18.0 \pm 7.5 \mathrm{kpc}$, which is a significant improvement over the constraint without the Pal 5 or GD-1 data (which was $22_{-9}^{+30} \mathrm{kpc}$ ). This improvement is due to the additional force measurements at $r \approx 14 \mathrm{kpc}$ and $r \approx 19 \mathrm{kpc}$, which allow the radial profile of the halo to be better determined. The mass of the dark-matter halo within $20 \mathrm{kpc}$ is also well constrained by these data: $M_{\text {halo }}(r<20 \mathrm{kpc})=1.1 \pm 0.1 \times 10^{11} \mathrm{M}_{\odot}$ (we define the mass as the average of $-r^{2} F_{r}$ at constant spherical $r=20 \mathrm{kpc}$ for the non-spherical dark-matter halos, but the exact definition is unimportant as we find the halo to be consistent with spherical). Such a halo mass and scale radius are fully consistent with the expected mass and concentration for the Milky Way's halo.

To investigate how these results depend on the assumed density distribution of the disk, we have repeated this fit for a double-exponential disk model without and with an additional gas component that is the same as that in $\S 2.2$. The best-fit value of the halo axis ratio in both cases is slightly, but not very significantly, lower: $c / a=0.99 \pm 0.14$ and $c / a=0.98 \pm 0.13$ for without and with gas, respectively; the $2 \sigma$ lower limit is $c / a=0.75$ in both cases. The mass within $20 \mathrm{kpc}$ and the scale radius of the halo are almost unchanged. The detailed mass profile of the disk therefore only has a minor effect. The scale length and height of the disk in all of the disk models, or $R_{0}$ and $V_{c}\left(R_{0}\right)$ are as expected not significantly better constrained after adding in the stream constraints.

As discussed in the introduction of this paper, we expect the Milky Way, with its massive, maximal disk (Bovy \& Rix 2013) to have $c / a \approx 0.8$. This is almost $2 \sigma$ below our best value for $c / a$ (the $2 \sigma$ lower limit is $c / a=0.79)$. Thus, our finding that the halo is spherical is in tension with the predictions from numerical simulations. Better data on the Pal 5 and GD-1 streams from, e.g., Gaia or similar data on additional streams will allow us in the near future to better determine whether this tension is real or not.

To summarize current measurements of the force field in the inner Milky Way, we display the force field in the best-fit model in Figure 10. We include direct measurements of the forces from the Pal 5 and GD-1 streams from this paper to demonstrate how they agree with those derived from the joint fit. We also include a summary of the results on the rotation curve (Bovy et al. 2012) and vertical-force curve (Bovy \& Rix 2013). Because these are not measured at the same location (the rotation curve being measured at $Z=0$ and the vertical force measured at $|Z|=1.1 \mathrm{kpc}$ ), we move the radial-force measurements from the rotation curve to $|Z|=1.1 \mathrm{kpc}$ using the fact that $\partial F_{R} / \partial Z=\partial F_{Z} / \partial R$ (Bovy \& Tremaine 2012) and assuming the $\partial F_{Z} / \partial R$ value measured by Bovy \& Rix (2013). While there remains a large volume to be explored, this figure clearly demonstrates the improvement in our understanding of the halo's potential and its shape provided by the Pal 5 and GD-1 force measurements.

\section{CONCLUSION}

In this paper we have performed the first proper stream fits to observational data using the action-angle approach to modeling stellar streams (e.g., Eyre \& Binney 2011; Bovy 2014; Sanders 2014). This is also the first analysis to combine data from multiple streams to improve our knowledge of the Milky Way's gravitational potential within $20 \mathrm{kpc}$ from the center.

Comparing our fits to previous analyses of the same (for GD-1) or similar (for Pal 5) data, we note that our analysis is much faster while simultaneously exploring a wider range of potentials than that considered by Bowden et al. (2015) for GD-1 and Küpper et al. (2015) for Pal 5. Because the action-angle approach directly provides a smooth stream track that can be computed using $\approx 60$ orbit integrations per model, we can evaluate the likelihood of different models in tens of seconds. This is the case, even though we are modeling the dark-matter halo using the flattened density of equation (1), for which computing the potential and forces is non-trivial and requires numerical integration. By approximating the stream as a single orbital torus over the observable, high-surface brightness part of the stream, we also keep the number of parameters the same as in the orbit fit of GD-1 by Koposov et al. (2010) (for a single of our 32 potential families), which means that our fit explores a volume of the same dimensionality as in the simple orbit approximation, while properly fitting a stream. Thus, we are able to fit the stream without resorting to high-performance computing and are thus able to easily fit multiple streams.

By exploring a wide range of potential families for both the Pal 5 and GD-1 streams, we have shown that the only information gained about the Milky Way's gravitational potential appears to be the local forces. This is because we can fit these streams equally well for all a priori likely potentials that explore a wide range of disk-to-halo ratios, disk profiles, and halo radial profiles. However, in each of 32 potential families that we consider for each stream, the a posteriori probable forces in the vicinity of the stream are the same. Despite this, we find that the form of the potential does matter in the fit, because comparing the results of our three-component potential fits to a simple logarithmic-potential fit, we find tighter and somewhat different constraints on the local forces. This is due to the fact that our three-component potential families properly take into account the effect of the varying offset between the stream track and an orbit for different potentials, which is absent in the less realistic logarithmic potential.

For both the Pal 5 and GD-1 streams, we find that the ratio of the vertical-to-radial components of the local force is more strongly constrained than their over- 
all amplitude. This is because, when fitting the phasespace data for both streams, an overall force-amplitude change can be traded off for differences in the distance or proper motion of the stream or progenitor (see Figures 2 and 6 ). Therefore, we express our constraints on the gravitational potential from both streams in terms of a measurement of the potential flattening $q_{\Phi}$ on the one hand and in terms of a constraint on the force vector projected onto the direction of constant flattening. These constraints are given in equations (4) and (5) for Pal 5 and equations (6) and (7) for GD-1. All of these constraints have divided out the effective prior due to the potential families used in the fit and are therefore pure measurements based on the data for Pal 5 and GD1 alone. The new force measurements are summarized in Figure 10, which demonstrates that they agree very well with the overall best-fit force field to a variety of dynamical data in addition to the stream data from this paper.

Because the streams considered here are exquisitely sensitive to the flattening of the potential, they provide a strong constraint on the axis ratio $c / a$ of the darkmatter halo's density. For GD- 1 and Pal 5 individually we measure $c / a=1.3_{-0.3}^{+0.5}$ for GD- $1(r \approx 14 \mathrm{kpc})$ and $c / a=0.93 \pm 0.16$ for Pal $5(r \approx 19 \mathrm{kpc})$. The GD-1 constraint is weaker because the stream is located much closer to the disk; the Pal 5 constraint is especially stringent because at its $(R, Z) \approx(8.4,16.8) \mathrm{kpc}$ location, the potential is almost entirely dominated by the darkmatter halo and the overall potential flattening that we measure $\left(q_{\Phi}=0.94 \pm 0.05\right)$ is almost directly that of the halo. While the estimates are consistent, the radial trend is towards a more oblate halo further out.

The combined constraint from $\mathrm{Pal} 5$ and GD-1 is $c / a=1.05 \pm 0.14$. This measurement of the halo axis ratio is obtained in the context of a three-component potential model for the Milky Way, but displays no correlations with the other parameters describing the potential. This result is at odds with the misaligned, triaxial halo at $20 \mathrm{kpc}<r<60 \mathrm{kpc}$ found to describe the kinematics of the Sgr stream well (Law \& Majewski 2010). From numerical simulations including the effect of growing a massive baryonic disk, we expect the Milky Way to have $c / a \approx 0.8$, which is in $\approx 2 \sigma$ tension with our measurement. While the tension is too mild currently to strongly disfavor the standard collisionless cold dark matter model for the formation of dark matter halos, our result hints at the importance of processes that sphericalize the Milky Way halo.

We also measure the total mass of the halo within 20 kpc to be $M_{\text {halo }}(r<20 \mathrm{kpc})=1.1 \pm 0.1 \times 10^{11} \mathrm{M}_{\odot}$ with a scale radius of $18.0 \pm 7.5 \mathrm{kpc}$. Such a halo mass and scale radius are fully consistent with the expected mass and concentration for the Milky Way's halo.

The available data on tidal streams will soon increase in both quantity and quality with the release of the Gaia data. Especially the precise, sub mas $\mathrm{yr}^{-1}$ proper motion measurements for the brightest stream members will significantly improve the measurements of the local force from these streams (see Figure 5 and Figure 8). It will also increase the amount of data on other, more distant streams like the Orphan stream and potentially wholly new streams. The methodology of the B14 stream model

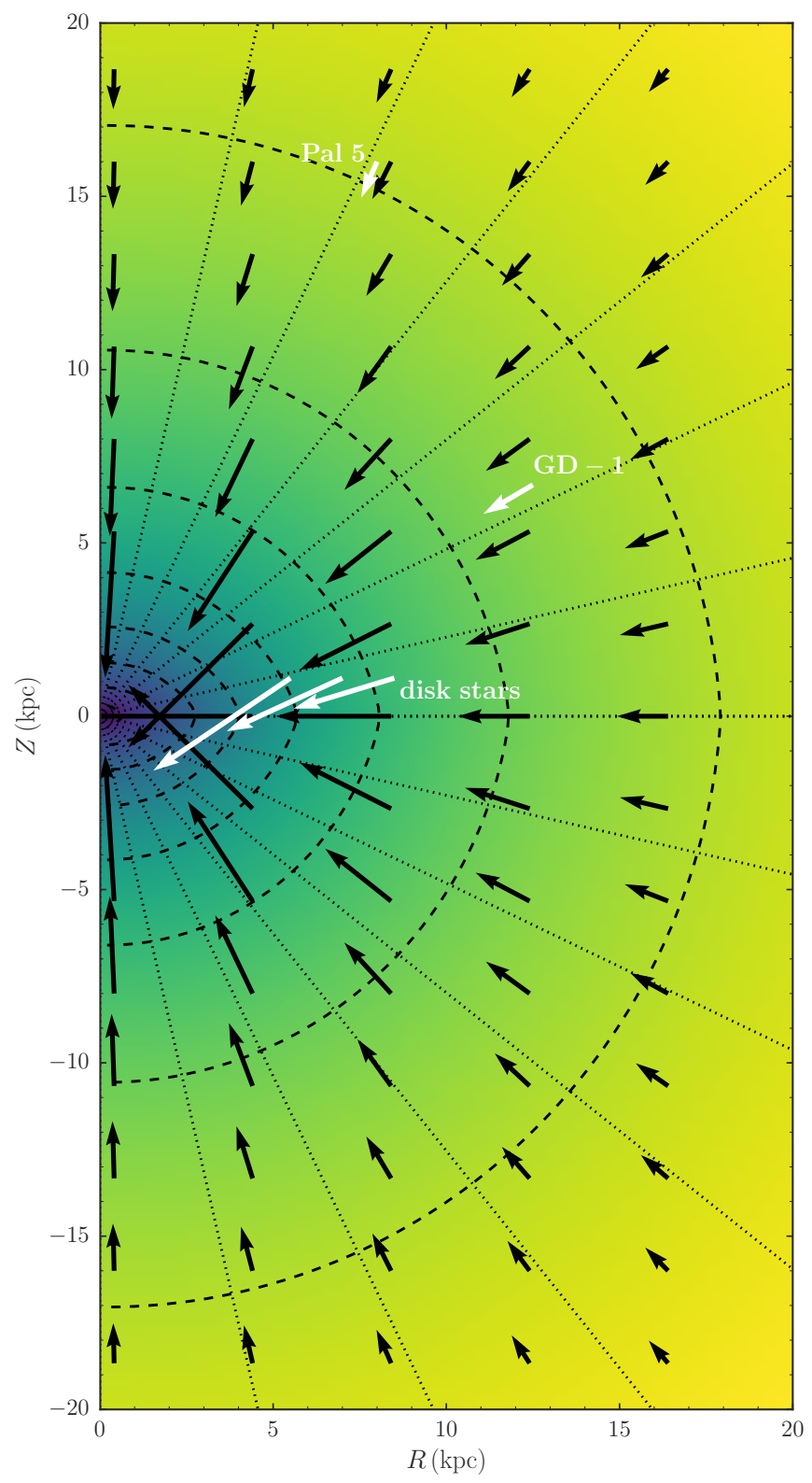

FIG. 10.- Best-fit force field for the Milky Way. The vectors display the two-dimensional radial and vertical components of the force field in the best-fit model to the data in $\S 2.2$ and the data derived from the Pal 5 and GD-1 streams in this paper. The color map and dashed contours show the value of the gravitational potential itself. The dotted lines go radially outward from the Galactic center to indicate the direction of a force pointing towards the center. The white vectors are direct measurements of the forces: the combination of the radial and vertical forces from Bovy et al. (2012) and Bovy \& Rix (2013) (labeled "disk stars" and summarized into three vectors), the force at the location of the Pal 5 cluster from $\S 4$, and the force at the location of the GD-1 stream from $\S 5$.

as used in this paper will then allow fast, flexible fits to all of the different streams that can be combined to further build up our direct measurements of the force field as in Figure 10 and be interpreted in terms of disk and halo models. Because steady-state modeling of the smooth halo will also allow tight constraints on the gravitational forces in the same volume, a comparison between the results from steady-state modeling - sensitive to the current forces - and those from streams - affected by the forces over the entire lifetime of the stream-may also 
allow direct inferences about the growth of the Milky Way's dark-matter halo (see, e.g., Buist \& Helmi 2015). All code used in this paper is made publicly available at

http://github.com/jobovy/mwhalo-shape-2016 .

We thank Naomi McClure-Griffiths for providing the new HI terminal velocity measurements from McClure-
Griffiths \& Dickey (2016) in electronic form and the anonymous referee for a constructive report. JB and $\mathrm{AB}$ received support from the Natural Sciences and Engineering Research Council of Canada. JB also received partial support from an Alfred P. Sloan Fellowship. TF \& NK are supported by the NSF CAREER award 1455260. The MCMC analyses in this work were run using emcee (Foreman-Mackey et al. 2013).

\section{REFERENCES}

Abadi, M. G., Navarro, J. F., Fardal, M., Babul, A., \& Steinmetz, M. 2010, MNRAS, 407, 435

Amorisco, N. C. 2015, MNRAS, 450, 575

Binney, J. 2013, New Astron. Rev., 57, 29

Binney, J., \& McMillan, P. J. 2016, MNRAS, 456, 1982

Binney, J., \& Piff, T. 2015, MNRAS, 454, 3653

Binney, J. \& Tremaine, S. 2008, Galactic Dynamics: Second Edition

Bland-Hawthorn, J., \& Gerhard, O. 2016, ARA\&A, 54, 529

Boehle, A., Ghez, A. M., Schödel, R., et al. 2016, ApJ, 830, 17

Bovy, J. 2014, ApJ, 795, 95 (B14)

Bovy, J. 2015, ApJS, 216, 29

Bovy, J., \& Rix, H.-W. 2013, ApJ, 779, 115

Bovy, J., \& Tremaine, S. 2012, ApJ, 756, 89

Bovy, J., Allende Prieto, C., Beers, T. C., et al. 2012, ApJ, 759, 131

Bovy, J., Nidever, D. L., Rix, H.-W., et al. 2014, ApJ, 790, 127

Bovy, J., Bird, J. C., García Pérez, A. E., et al. 2015, ApJ, 800, 83

Bovy, J., Erkal, D., \& Sanders, J. L. 2016, MNRAS, submitted (arXiv:1606.03470)

Bowden, A., Belokurov, V., \& Evans, N. W. 2015, MNRAS, 449, 1391

Bowden, A., Evans, N. W., \& Williams, A. A. 2016, MNRAS, 460, 329

de Bruijne, J. H. J. 2012, Ap\&SS, 341, 31

Buist, H. J. T., \& Helmi, A. 2015, A\&A, 584, A120

Carlberg, R. G., Grillmair, C. J., \& Hetherington, N. 2012, ApJ, 760,75

Chatzopoulos, S., Fritz, T. K., Gerhard, O., et al. 2015, MNRAS, 447, 948

Clemens, D. P. 1985, ApJ, 295, 422

Cole, S., \& Lacey, C. 1996, MNRAS, 281, 716

Davé, R., Spergel, D. N., Steinhardt, P. J., \& Wandelt, B. D. 2001, ApJ, 547, 574

Debattista, V. P., Moore, B., Quinn, T., et al. 2008, ApJ, 681, 1076

Dehnen, W. \& Binney, J. 1998, MNRAS, 294, 429

Dotter, A., Sarajedini, A., \& Anderson, J. 2011, ApJ, 738, 74

Dubinski, J., \& Carlberg, R. G. 1991, ApJ, 378, 496

Dubinski, J. 1994, ApJ, 431, 617

Eyre, A., \& Binney, J. 2011, MNRAS, 413, 1852

Fardal, M. A., Huang, S., \& Weinberg, M. D. 2015, MNRAS, 452, 301

Flynn, C., Holmberg, J., Portinari, L., Fuchs, B., \& Jahreiß, H. 2006, MNRAS, 372, 1149

Foreman-Mackey, D., Hogg, D. W., Lang, D., \& Goodman, J. 2013, PASP, 125, 306

Frenk, C. S., White, S. D. M., Davis, M., \& Efstathiou, G. 1988, ApJ, 327, 507

Fritz, T. K., \& Kallivayalil, N. 2015, ApJ, 811, 123

Gnedin, O. Y., Gould, A., Miralda-Escudé, J., \& Zentner, A. R. 2005, ApJ, 634, 344

Grillmair, C. J., \& Dionatos, O. 2006, ApJ, 643, L17

Gustafsson, M., Fairbairn, M., \& Sommer-Larsen, J. 2006 , Phys. Rev. D, 74, 123522

Harris, W. E. 1996, AJ, 112, 1487

Helmi, A., \& White, S. D. M. 1999, MNRAS, 307, 495

Holmberg, J. \& Flynn, C. 2000, MNRAS,313, 209
Ibata, R. A., Lewis, G. F., Irwin, M. J., \& Quinn, T. 2002, MNRAS, 332, 915

Ibata, R. A., Lewis, G. F., \& Martin, N. F. 2016, ApJ, 819, 1

Jing, Y. P., \& Suto, Y. 2002, ApJ, 574, 538

Johnston, K. V. 1998, ApJ, 495, 297

Johnston, K. V., Spergel, D. N., \& Haydn, C. 2002, ApJ, 570, 656

Kazantzidis, S., Abadi, M. G., \& Navarro, J. F. 2010, ApJ, 720, L62

Koposov, S. E., Rix, H.-W., \& Hogg, D. W. 2010, ApJ, 712, 260

Küpper, A. H. W., Balbinot, E., Bonaca, A., et al. 2015, ApJ, 803, 80

Kuzma, P. B., Da Costa, G. S., Keller, S. C., \& Maunder, E. 2015, MNRAS, 446, 3297

Law, D. R., \& Majewski, S. R. 2010, ApJ, 714, 229

Loebman, S. R., Ivezić, Ż., Quinn, T. R., et al. 2014, ApJ, 794, 151

McClure-Griffiths, N. M. \& Dickey, J. M. 2007, ApJ, 671, 427

McClure-Griffiths, N. M. \& Dickey, J. M. 2016, ApJ, 831, 124

McKee, C. F., Parravano, A., \& Hollenbach, D. J. 2015, ApJ, 814, 13

Merritt, D., \& Fridman, T. 1996, ApJ, 460, 136

Miralda-Escudé, J. 2002, ApJ, 564, 60

Navarro, J. F., Frenk, C. S., \& White, S. D. M. 1997, ApJ, 490, 493

Odenkirchen, M., Grebel, E. K., Rockosi, C. M., et al. 2001, ApJ, 548, L165

Odenkirchen, M., Grebel, E. K., Kayser, A., Rix, H.-W., \& Dehnen, W. 2009, AJ, 137, 3378

Olling, R. P., \& Merrifield, M. R. 2000, MNRAS, 311, 361

Pearson, S., Küpper, A. H. W., Johnston, K. V., \& Price-Whelan, A. M. 2015, ApJ, 799, 28

Peter, A. H. G., Rocha, M., Bullock, J. S., \& Kaplinghat, M. 2013, MNRAS, 430, 105

Price-Whelan, A. M., Hogg, D. W., Johnston, K. V., \& Hendel, D. 2014, ApJ, 794, 4

Rix, H.-W., \& Bovy, J. 2013, A\&A Rev., 21, 61

Sanders, J. L., \& Binney, J. 2013, MNRAS, 433, 1813

Sanders, J. L., \& Binney, J. 2013, MNRAS, 433, 1826

Sanders, J. L. 2014, MNRAS, 443, 423

Schönrich, R., Binney, J., \& Dehnen, W. 2010, MNRAS, 403, 1829

Spergel, D. N., \& Steinhardt, P. J. 2000, PRL, 84, 3760

Thomas, G. F., Ibata, R., Famaey, B., Martin, N. F., \& Lewis, G. F. 2016, MNRAS, 460, 2711

Tremaine, S. 1999, MNRAS, 307, 877

Varghese, A., Ibata, R., \& Lewis, G. F. 2011, MNRAS, 417, 198

Vivas, A. K., \& Zinn, R. 2006, AJ, 132, 714

Vera-Ciro, C. A., Sales, L. V., Helmi, A., et al. 2011, MNRAS, 416, 1377

Warren, M. S., Quinn, P. J., Salmon, J. K., \& Zurek, W. H. 1992, ApJ, 399, 405

Wegg, C., Gerhard, O., \& Portail, M. 2016, MNRAS, 463, 557

Williams, A. A., Evans, N. W., \& Bowden, A. D. 2014, MNRAS, 442,1405

Xue, X. X., Rix, H.-W., Zhao, G., et al. 2008, ApJ, 684, 1143

Yoshida, N., Springel, V., White, S. D. M., \& Tormen, G. 2000, ApJ, 535, L103

Zhang, L., Rix, H.-W., van de Ven, G., Bovy, J., Liu, C., Zhao, G. 2013, ApJ, 772, 108 